ORIGINAL PAPER

\title{
EGFR/PI3 K/AKT/MTOR PATHWAY IN HEAD AND NECK SQUAMOUS CELL CARCINOMA PATIENTS WITH DIFFERENT HPV STATUS
}

\author{
Anna Janecka-Widea ${ }^{1}$, Kaja Majchrzyk ${ }^{2}$, Anna Mucha-Maeecka ${ }^{2}$, Beata Biesaga ${ }^{3}$
}

\begin{abstract}
${ }^{1}$ Department of Tumor Pathology, Maria Sklodowska-Curie National Research Institute of Oncology, Krakow Branch, Poland ${ }^{2}$ Department of Radiotherapy, Maria Sklodowska-Curie National Research Institute of Oncology, Krakow Branch, Poland ${ }^{3}$ Center for Translational Research and Molecular Biology of Cancer, Maria Sklodowska-Curie National Research Institute of Oncology, Gliwice Branch, Poland
\end{abstract}

The aim of the study was to compare prognostic potential of $P I K 3 C A$ mutations and expression of proteins involved in or regulate EGFR/PI3K/Akt/mTOR signaling in HPV 16 positive and HPV negative head and neck squamous cell carcinoma (HNSCC) patients.

The expression of proteins (EGFR, Akt, pAkt(Ser473), pAkt(Thr308), mTOR, PTEN, pPTEN, APOBEC3B) were assessed immunohistochemically and PIK3CA mutations (p.E542K, p.E545K, p.H1047R) by qPCR.

Significantly more HPV16 positive tumors (89.29\%) with low EGFR expression were found as compared to HPV negative ones (58.82\%). PIK3CA mutations were detected in $7.14 \%$ of HPV16 positive and $2.5 \%$ of HPV negative cancers. In HPV16 positive patients survival analysis has shown that positive prognostic potential for disease free survival (DFS) had low expression of APOBEC3B. In HPV negative patients prognostic significance for DFS had APOBEC3B, Akt and pAkt(Thr308) levels, and for overall survival (OS) - pAkt(Thr308) only. Independent favorable prognostic factors in the whole group of patients were: low T stage, low pAkt(Thr308) expression, active HPV16 infection (for OS and DFS) and female gender (for OS).

Obtained results suggest the existence of significant differences in expression and prognostic potential of proteins involved in EGFR/PI3K/Akt $/ \mathrm{mTOR}$ signaling between HPV16 positive and HPV negative HNSCC patients.

Key words: HNSCC, active HPV infection, EGFR/PI3K/Akt/mTOR pathway, survival.

\section{Introduction}

The risk factors of head and neck squamous cell carcinoma (HNSCC) development are tobacco use, alcohol consumption and HPV infection. The HPV positive HNSCC is now established as a separate disease with distinct clinical (younger age, higher social status, low levels of alcohol consumption and ciga- rette smoking, association with high-risk sexual behaviors) and molecular (for example lack of TP53 and CDKN2A mutations) characteristics compared to HPV negative ones [1]. It was also proved that HNSCC patients with tumors related to HPV infection had increased survival after surgical treatment [2], radiotherapy [3] and combined treatment approaches [4] compared to HPV negative ones. 
HPV oncogenic activity is related mainly to E6 and E7 viral oncoproteins. E6 initiates degradation of $\mathrm{p} 53$ - the tumor suppressor protein involved in regulation of cell cycle, DNA repair and cell death. $\mathrm{E} 7$ binds to $\mathrm{Rb}$ protein, resulting in lack of G1 checkpoint activity [5]. Viral proteins not only inhibit the tumor suppressors $\mathrm{p} 53$ and $\mathrm{Rb}$, but also alter other signaling cascades that may play important role in carcinogenesis and response to ionizing radiation or drugs, such as EGFR/PI3K/Akt/mTOR pathway.

In healthy cells signal begins from the activation of receptor tyrosine kinases family, such as epidermal growth factor receptor (EGFR). EGFR activates phosphatidylinositol 3-kinase (PI3K), leading to its allosteric activation and tyrosine phosphorylation of its regulatory subunit. Activation of PI3K results in phosphorylation of the key effector protein kinase Akt. Active Akt regulates multiple signaling pathways that maintain cell cycle, proliferation and apoptosis. It phosphorylates downstream targets, including mammalian target of rapamycin (mTOR), which plays a central regulating role in protein synthesis, metabolism and cell growth. Phosphatase and tensin homologue deleted on chromosome ten (PTEN), on the other hand, is a key negative regulator of EGFR/ $\mathrm{PI} 3 \mathrm{~K} / \mathrm{Akt} / \mathrm{m}$ TOR signaling [6].

During carcinogenesis overactivation of EGFR/ $\mathrm{PI} 3 \mathrm{~K} / \mathrm{Akt} / \mathrm{mTOR}$ cascade is often observed. It leads to uncontrolled growth, angiogenesis, metastatic potential and therapy resistance [7]. There are some experimental and preclinical data $[8,9,10,11,12]$, which suggest that HPV disrupts EGFR/PI3K/Akt/ mTOR pathway through both genes mutations and changes in protein expression of pathway components. HPV associated proteins (mainly E6 and E7) may deregulate EGFR/PI3K/Akt/mTOR functioning directly through influence on the expression of signal transmitters as well as indirectly - activating the apolipoprotein $\mathrm{B}$ mRNA editing enzyme catalytic subunit 3B (APOBEC3B). This enzyme catalyzes reaction of cytosine deamination resulting in the conversion of cytosine to uracil and therefore, is a source of multiple mutations in cells including mutations of PIK3CA gene, which encodes catalytic subunit of PI3K enzyme [9, 10].

Although there are many reports that have examined EGFR, Akt, PTEN, mTOR and APOBEC3B expressions in HNSCC, there is no comprehensive study of all these markers together in the group of HNSCC patients. This prompted us to study the expression of selected proteins involved in EGFR/PI3K/Akt/ mTOR pathway and proteins regulate this cascade (EGFR, Akt, pAkt(Ser473), pAkt(Thr308), mTOR, PTEN, pPTEN, APOBEC3B) as well as the frequency of PIK $3 C A$ mutations in relation to HPV status in HNSCC patients. Moreover, we determined the relations between studied biomarkers and clinical and histopathological characteristics as well as analyzed their prognostic potential.

\section{Material and methods}

\section{Patients}

Formalin-fixed paraffin-embedded (FFPE) specimens were collected from 155 patients diagnosed with HNSCC (25 oral cavity, 66 oropharyngeal, 6 hypopharyngeal and 58 laryngeal squamous cell carcinoma cases), treated between 1991 and 2014 in Maria Sklodowska-Curie National Research Institute of Oncology, Cracow Branch. Detailed clinical and histopathological data were summarized in Table I. The level of smoking was calculated as number of cigarettes per day $\times$ years of smoking. The level of drinking was defined as 'low' for no/occasional alcohol drinkers or 'high' for alcoholics and people drink more than 15 drinks of high percentage alcohol per week. Additionally, status of active HPV16 infection (based on simultaneous assessment of immunohistochemical p16 overexpression and HPV16 DNA presence by nested PCR and qPCR) was assessed for every tumor [13]. For each FFPE, histopathological reverification was performed due to confirm squamous cell carcinoma diagnosis and to indicate blocks with at least $50 \%$ of tumor component for mutational and immunohistochemical (IHC) analyses.

\section{IHC staining}

The evaluation of EGFR, Akt, pAkt, mTOR, APOBEC3B, PTEN and pPTEN expressions was performed based on immunohistochemically stained FFPE tissue sections. All necessary details of staining procedure are presented in Table II. Briefly, sections were cut at $4 \mu \mathrm{m}$, mounted on SuperFrost Plus slides (Menzel-Gläser, Germany) and then deparaffinized and hydrated through a series of xylenes and alcohols. After antigen unmasking, slides were incubated for $10 \mathrm{~min}$ with peroxidase block and $30 \mathrm{~min}$ in $0.3 \% \mathrm{H}_{2} \mathrm{O}_{2}$ diluted in $100 \%$ methanol (to quench an exogenous peroxidases). Non-specific binding of antibodies was blocked during $5 \mathrm{~min}$ incubation with UltraVision Protein Block (Thermo Scientific, Fremont, USA). Next, incubation with primary antibody was performed and section were treated for 45 min with BrightVision Plus Poly-HRP-Anti $\mathrm{MS} / \mathrm{Rb} / \mathrm{Rt}$ IgG detection system (Immunologic, Duiven, The Netherlands) and DAB (3,3'-diaminobenzidine) (Vector Laboratories, Inc., Burlingame, USA). Hematoxylin was applied for nuclear counterstaining. Each step of IHC procedure was followed by washing in tris-buffered saline and Tween 20 (TBST). HNSCC tissues with high expression of wanted pro- 
$2=\frac{\sqrt{2}}{8}$

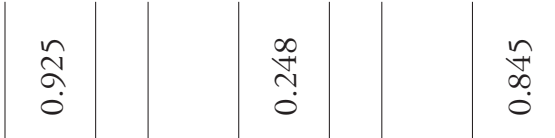

ลิํำ

亭

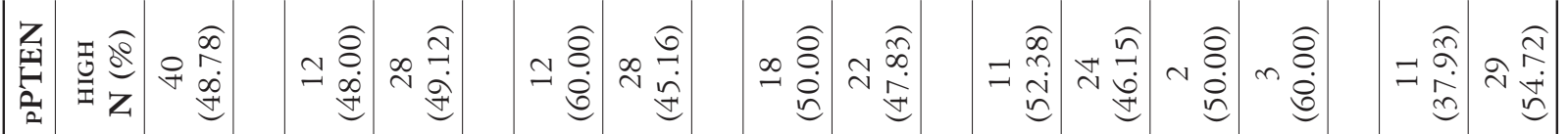

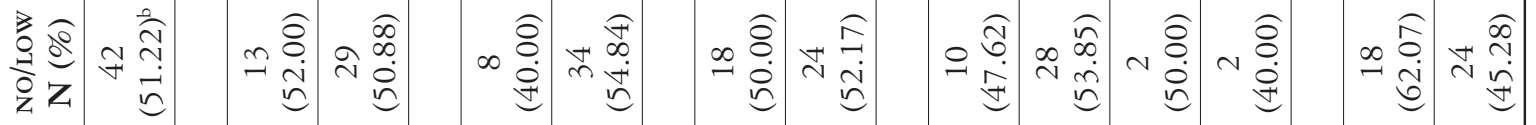

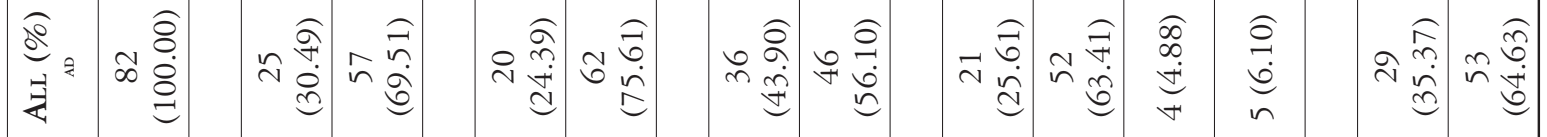

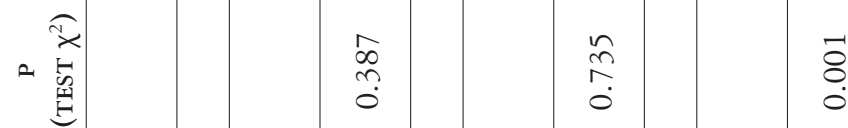

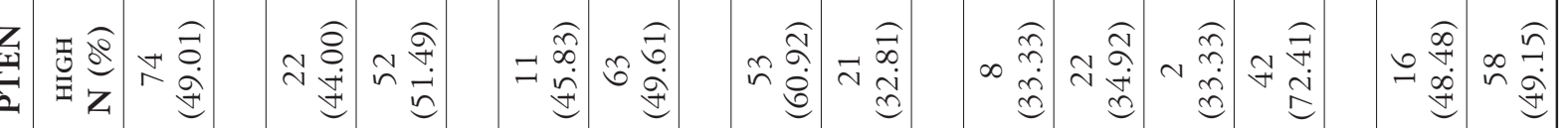

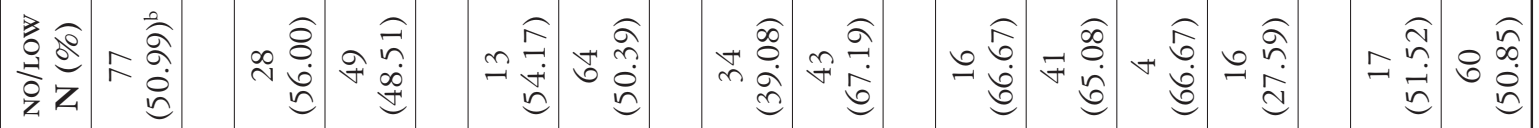

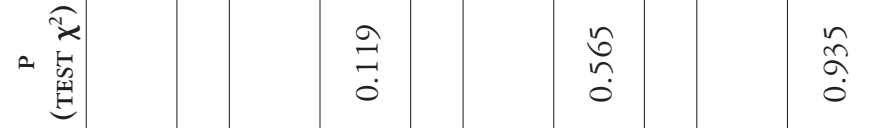

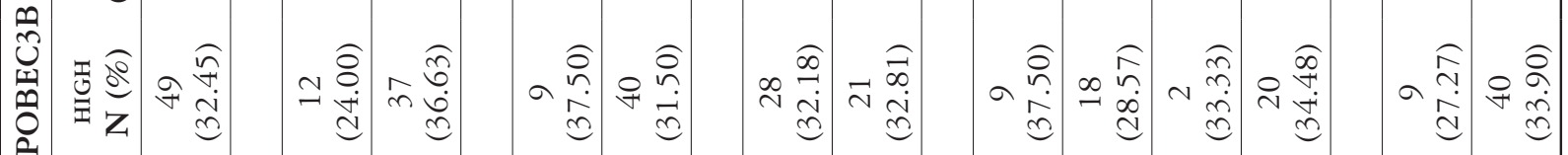

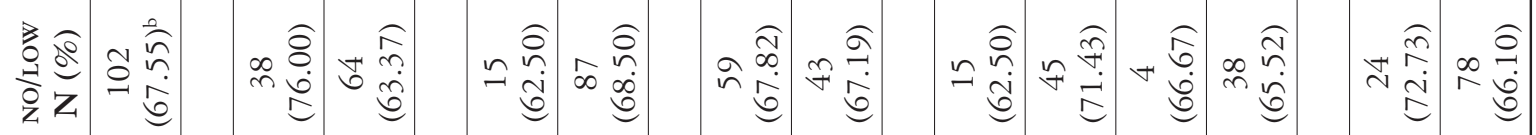

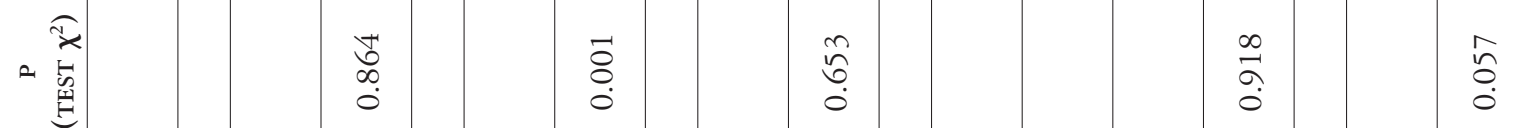

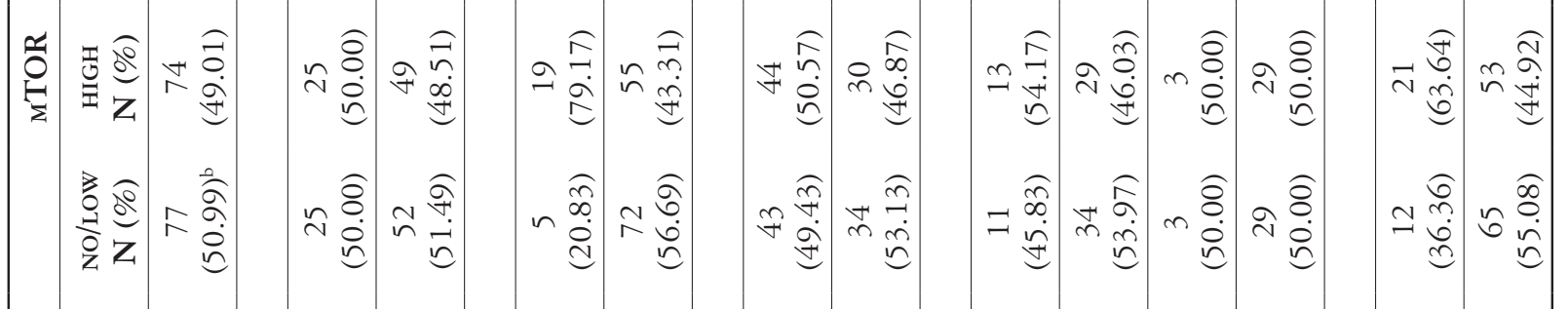

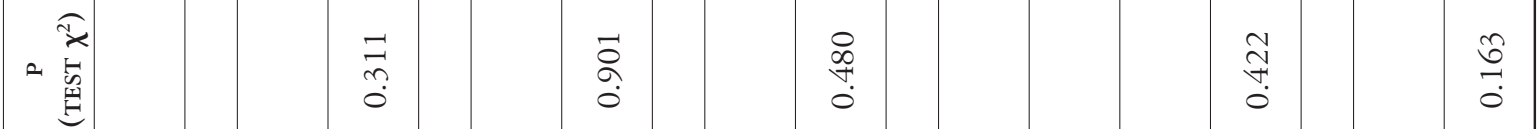

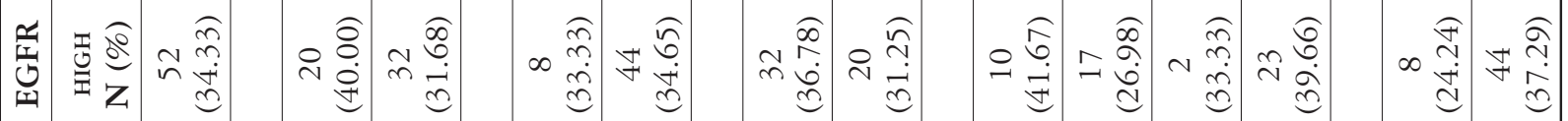

䓈

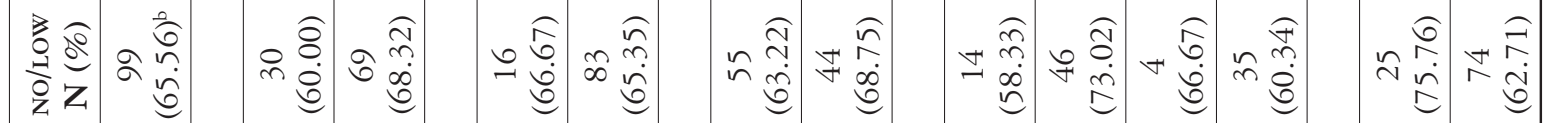

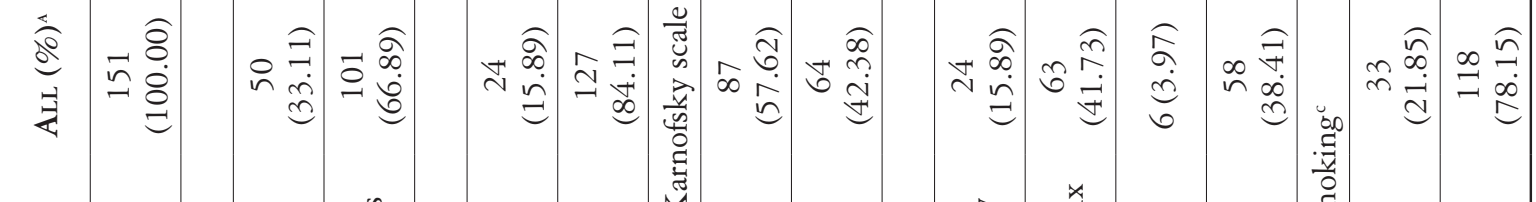

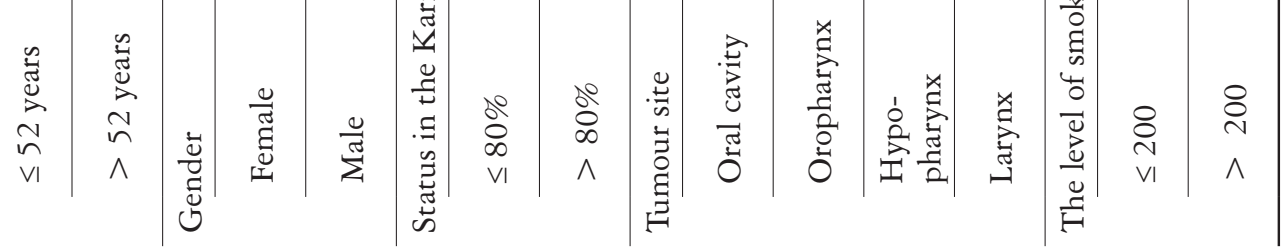




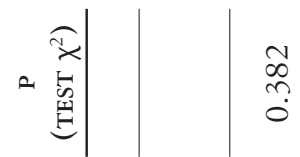

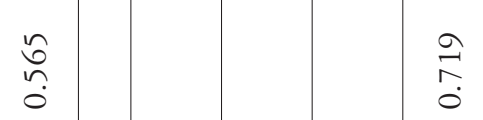

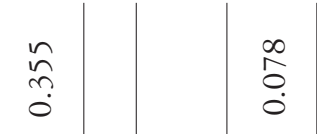

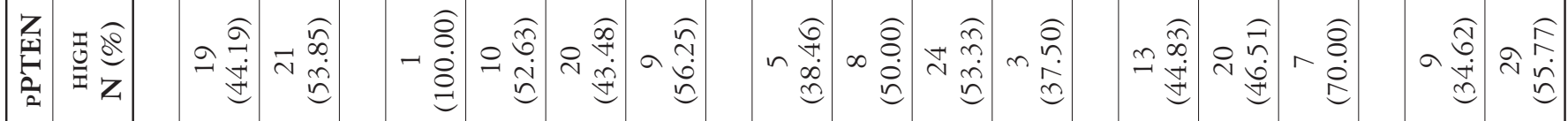

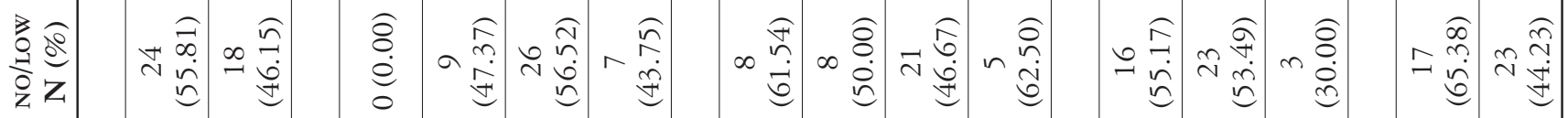

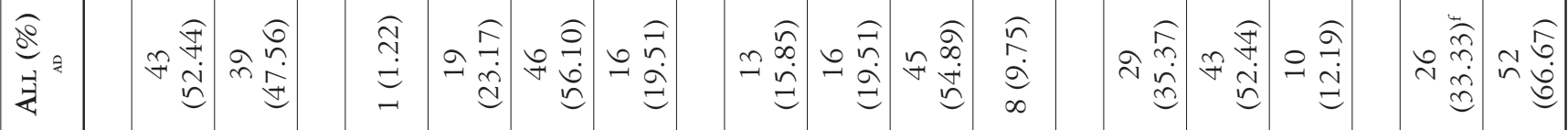

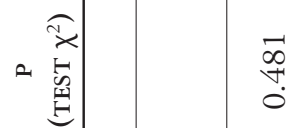

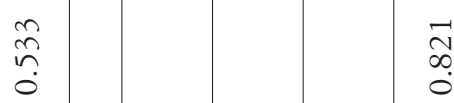

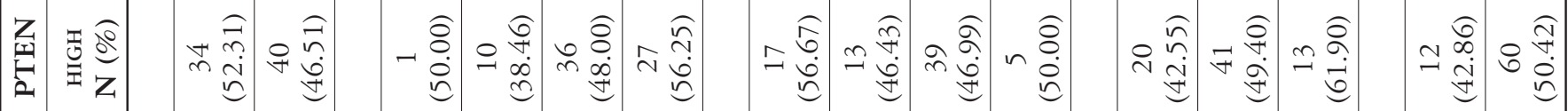

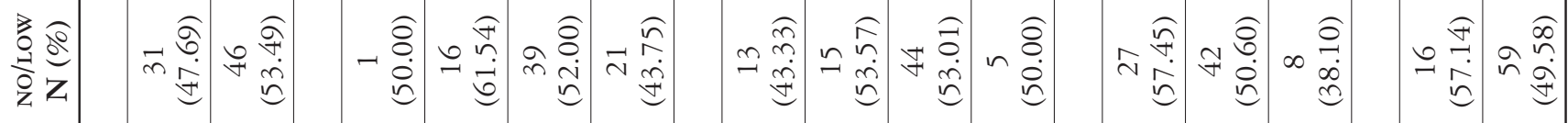

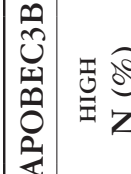

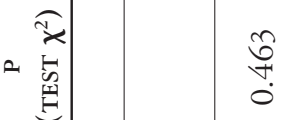

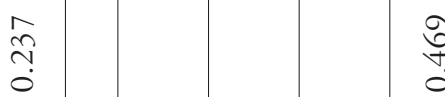

\begin{tabular}{l|l}
0 \\
0 \\
0
\end{tabular}

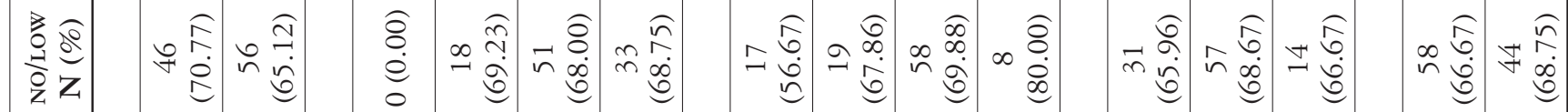

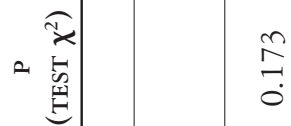

à

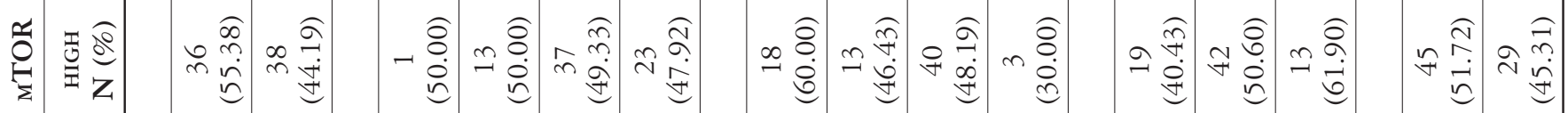

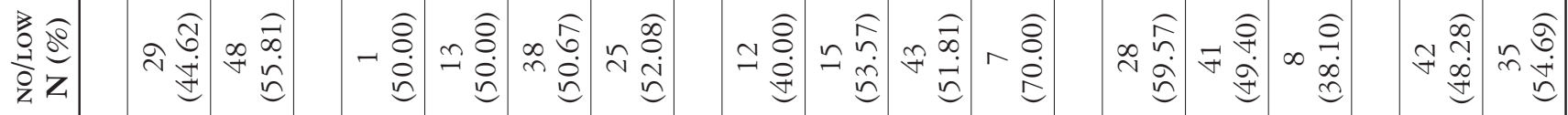

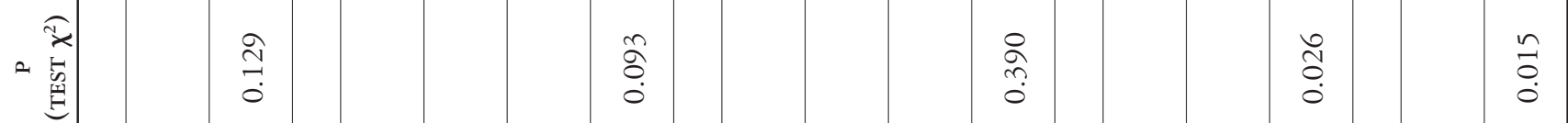

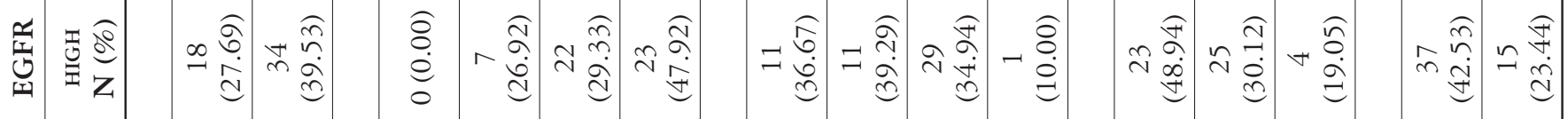

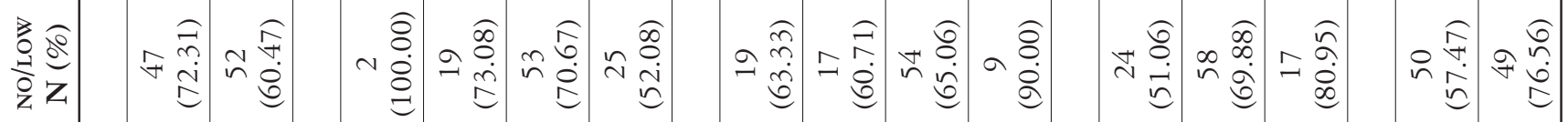

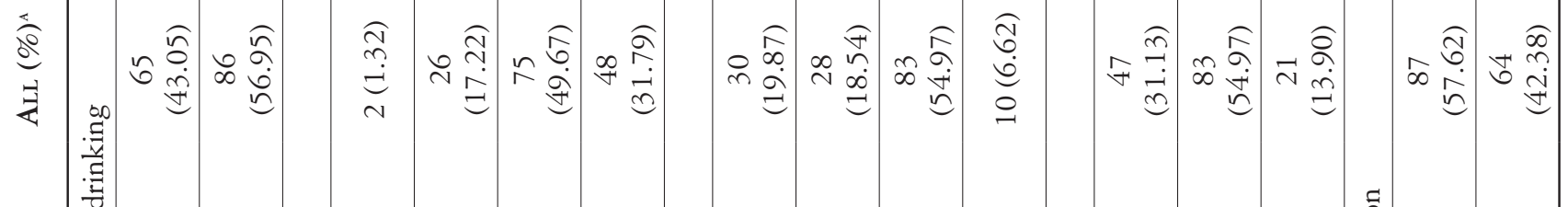

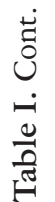

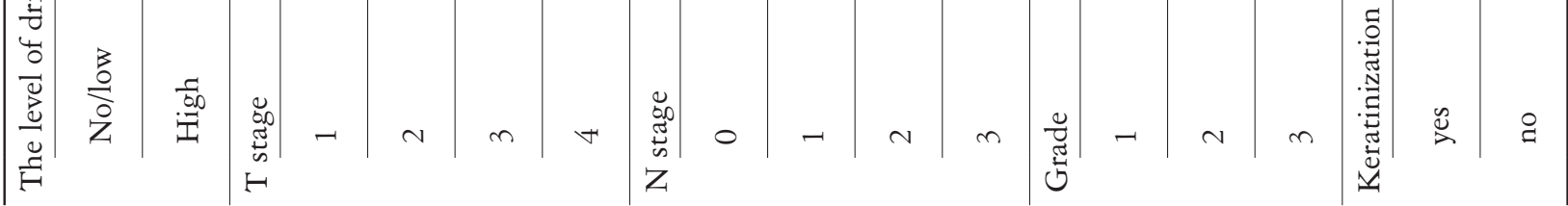




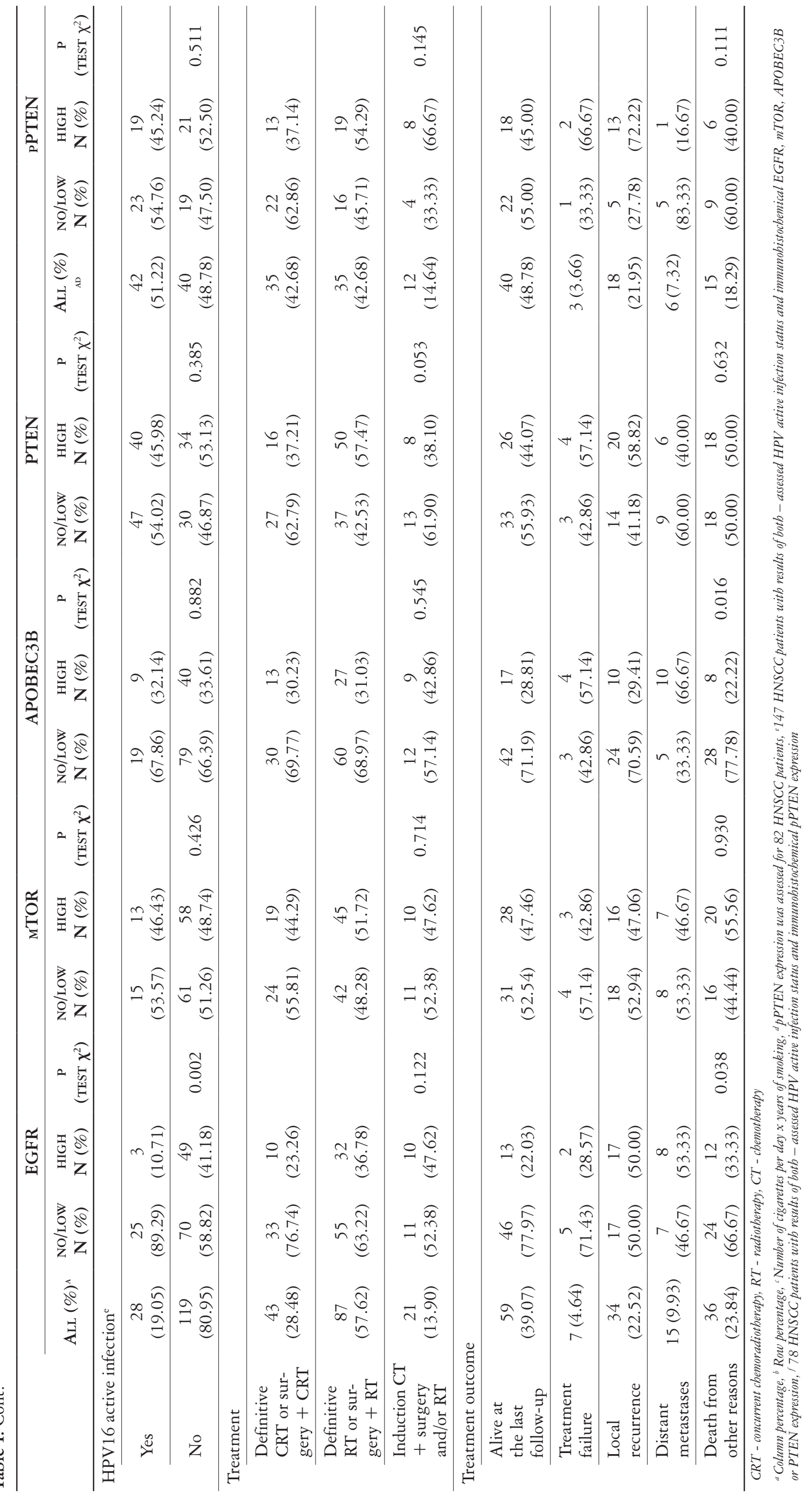


Table II. Details of immunohistochemical staining procedure

\begin{tabular}{|c|c|c|c|c|}
\hline AnTigen & Clone & MANUFACTURER & ANTIGEN RETRIEVAL & $\begin{array}{l}\text { INCUBATION WITH } \\
\text { PRIMARY ANTIBODY }\end{array}$ \\
\hline EGFR & H11 & $\begin{array}{c}\text { Dako Agilent Technologies } \\
\text { (Denmark A/S, Glostrup, } \\
\text { Denmark) }\end{array}$ & $\begin{array}{c}\text { Proteinase K } \\
\text { RT } 10 \mathrm{~min} \\
\text { (Agilent Dako S3020) }\end{array}$ & $\begin{array}{l}1: 200,4^{\circ} \mathrm{C} \\
\text { overnight }\end{array}$ \\
\hline Akt & C67E7 & $\begin{array}{l}\text { Cell Signaling Technology } \\
\text { (Danvers, USA) }\end{array}$ & $\begin{array}{l}\text { Target Retrieval Solution TRS: pH 6.1; } \\
\quad 96^{\circ} \mathrm{C} 1 \text { h (Agilent Dako S1699) }\end{array}$ & $\begin{array}{l}1: 150,4^{\circ} \mathrm{C} \\
\text { overnight }\end{array}$ \\
\hline pAkt(Thr308) & - & $\begin{array}{c}\text { Biorbyt Ldt. } \\
\text { (Cambridge, UK) }\end{array}$ & $\begin{array}{l}\text { Target Retrieval Solution TRS: pH 6.1; } \\
96^{\circ} 50 \text { min (Agilent Dako S1699) }\end{array}$ & $\begin{array}{l}1: 250,4^{\circ} \mathrm{C} \\
\text { overnight }\end{array}$ \\
\hline pAkt(Ser473) & D9E XP & $\begin{array}{l}\text { Cell Signaling Technology } \\
\text { (Danvers, USA) }\end{array}$ & $\begin{array}{l}\text { Target Retrieval Solution TRS: pH 6.1; } \\
\quad 96^{\circ} \mathrm{C} 1 \text { h (Agilent Dako S1699) }\end{array}$ & $\begin{array}{l}1: 30,4^{\circ} \mathrm{C} \\
\text { overnight }\end{array}$ \\
\hline mTOR & 7C10 & $\begin{array}{l}\text { Cell Signaling Technology } \\
\text { (Danvers, USA) }\end{array}$ & $\begin{array}{l}\text { Target Retrieval Solution TRS: pH 6.1; } \\
96^{\circ} 45 \text { min (Agilent Dako S1699) }\end{array}$ & $\begin{array}{l}1: 75,4^{\circ} \mathrm{C} \\
\text { overnight }\end{array}$ \\
\hline PTEN & 138G6 & $\begin{array}{l}\text { Cell Signaling Technology } \\
\text { (Danvers, USA) }\end{array}$ & $\begin{array}{l}\text { Target Retrieval Solution TRS: pH 6.1; } \\
96^{\circ} 50 \text { min (Agilent Dako S1699) }\end{array}$ & $1: 75,1 \mathrm{~h}$ at $37^{\circ} \mathrm{C}$ \\
\hline $\begin{array}{l}\text { pPTEN } \\
(\text { Ser380) }\end{array}$ & - & $\begin{array}{l}\text { Invitrogen Thermo Fisher } \\
\text { Scientific (Fremont, CA, USA) }\end{array}$ & $\begin{array}{l}\text { Target Retrieval Solution TRS: pH 6.1; } \\
96^{\circ} 50 \text { min (Agilent Dako S1699) }\end{array}$ & $\begin{array}{l}1: 50,4^{\circ} \mathrm{C} \\
\text { overnight }\end{array}$ \\
\hline AРОВЕС3В & - & $\begin{array}{c}\text { Biorbyt Ldt } \\
\text { (Cambridge, UK) }\end{array}$ & $\begin{array}{l}\text { Target Retrieval Solution TRS: pH 6.1; } \\
96^{\circ} 20 \text { min (Agilent Dako S2367) }\end{array}$ & $\begin{array}{l}1: 250,4^{\circ} \mathrm{C} \\
\text { overnight }\end{array}$ \\
\hline
\end{tabular}

tein were used as positive controls. The primary antibodies were omitted for negative controls.

The complete results of protein expression data were obtained for 151 patients (for 4 patients there was not enough amount of material in the block), except for pPTEN where results only for 82 patients were possible to collect.

\section{IHC evaluation}

In general the staining intensity and percentage of stained tumor cells were assessed (Fig. 1). The staining intensity of EGFR, Akt, pAkt, mTOR and APOBEC $3 B$ was assessed as $0-$ no, 1 - weak, 2 - moderate or 3 - strong staining. In the case of PTEN and pPTEN the following scale was used: $0-$ no, $1-$ weak and 2 - strong staining. Stained sections were reviewed independently by 2 researchers. For all proteins $\mathrm{H}$-score was calculated according to the following formula: $\mathrm{H}$-score $=(1 \times \%$ of cells with staining intensity 1$)+(2 \times \%$ of cells with staining intensity 2$)+(3 \times \%$ of cells with staining intensity 3). We decided to use this method as the only one that takes into account heterogeneity of tumor.

For all assessed proteins we defined expression as 'high' - for tissues with $\mathrm{H}$-score higher than cut off value or 'low' - for tissues with H-score equal or lower than cut off value. We used the following cut off values: 148.5 for EGFR expression, 147.5 for Akt expression, 33 for $\mathrm{pAkt}(\operatorname{Ser} 473), 183$ for $\mathrm{pAk}$ -
t(Thr308), 180.0 for mTOR expression, 198.0 for APOBEC3B expression, 85.0 for PTEN expression and 107.5 for $\mathrm{PPTEN}$ expression.

\section{DNA isolation}

DNA isolation was performed using $5 \mu \mathrm{m}$ thick 3-5 sections cut from selected FFPE blocks. DNA was extracted manually using ReliaPrep FFPE gDNA Miniprep System form Promega Corp (Madison, WI, USA) according to manufacturer's protocol with one own modification (overnight instead of $1 \mathrm{~h}$ digestion at $56^{\circ} \mathrm{C}$ ). The purity (measured as A260/280 and A260/230 ratios) and concentration of DNA were evaluated spectrophotometrically with Biophotometer Plus (Eppendorf, Germany) with TrayCell (Hellma, Germany) according to manufacturer's suggestions. Samples were stored at $-20^{\circ} \mathrm{C}$ until used.

\section{PIK3CA mutational analyses}

To assess the PIK3CA mutational status, real-time polymerase chain reaction (qPCR) was performed using ViiA7 Real-Time PCR System (Applied Biosystems, Foster City, CA, USA). For each patient, 4 different assays were performed on the same plate: (1) PIK3CA_775 mu TaqMan Mutation Detection Assay (Assay ID : Hs00000831 mu) allowing for p.H1047R (c.3140A > G) detection, (2) PIK3CA $763 \mathrm{mu}$ TaqMan Mutation Detection Assay (Assay ID: Hs00000824_mu) allowing for p. E545K (c.1633G $>$ A) detection, (3) PIK- 
0
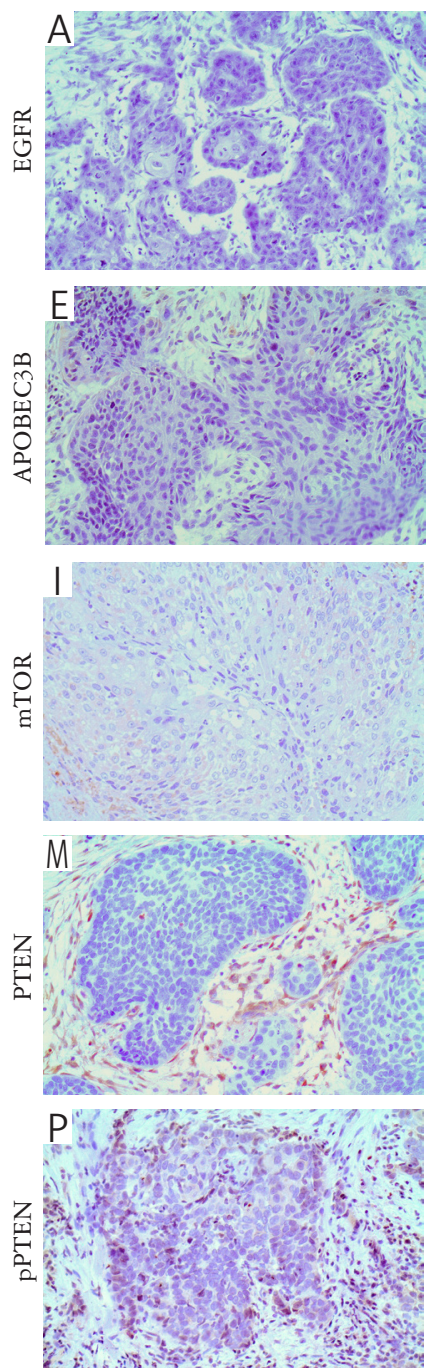

1
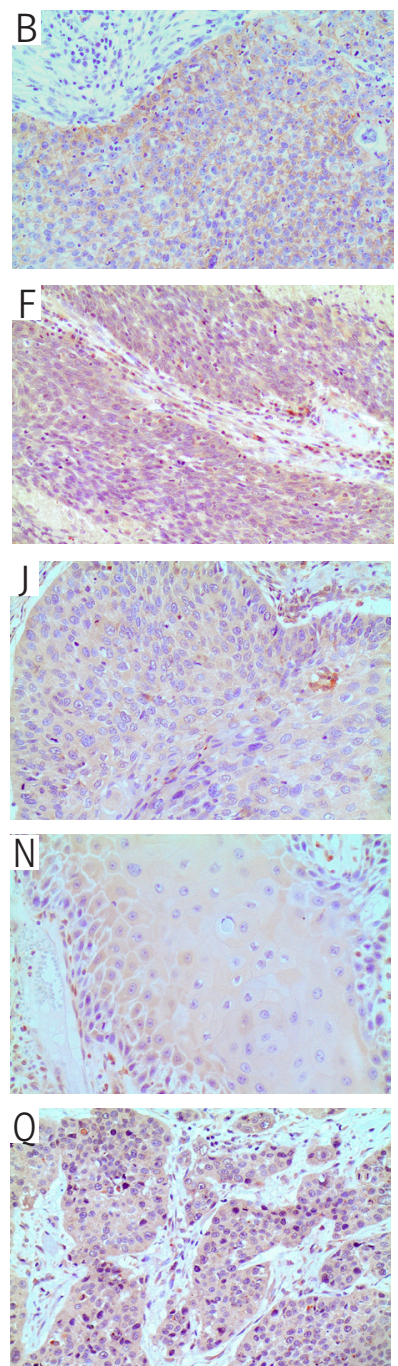

2
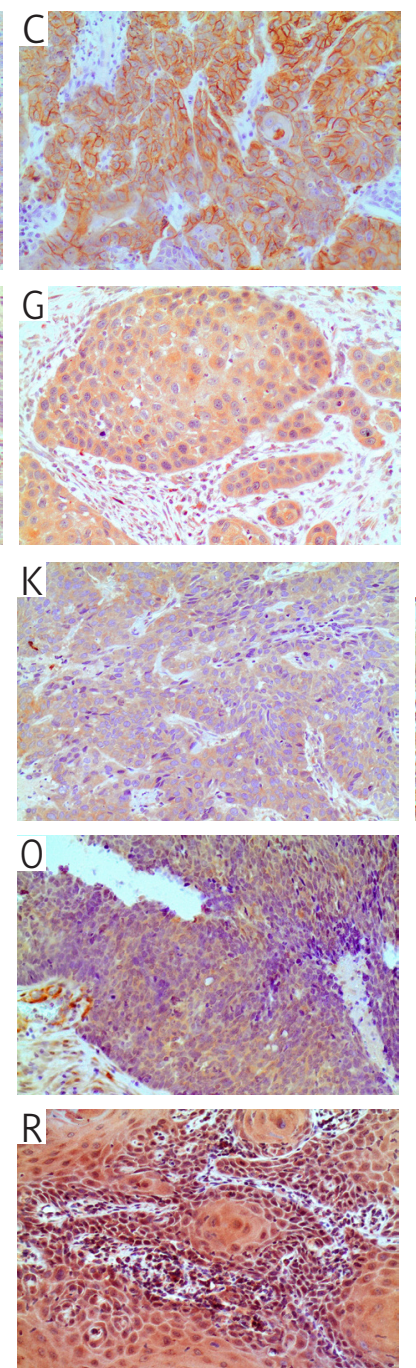

3
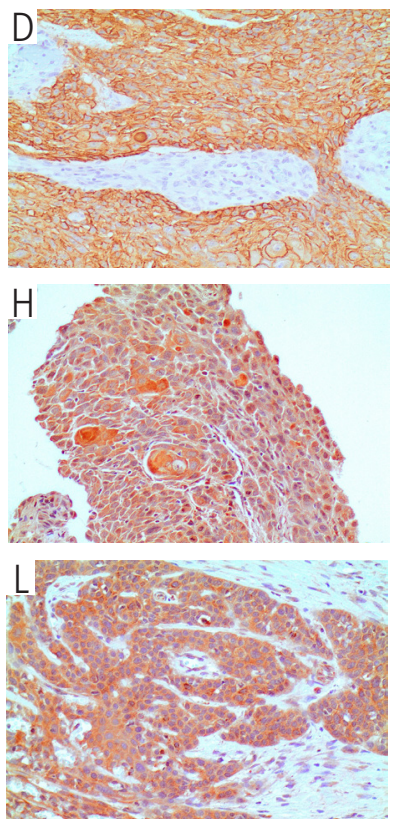

Fig. 1. Representative images of EGFR, APOBEC3B, mTOR, PTEN and PPTEN signal in HNSCC tissue based on immunohistochemical staining. Different staining intensity (for EGFR, APOBEC3B and mTOR: 0 - lack of staining, 1 - weak, 2 - moderate, 3 - strong; for PTEN and pPTEN: 0 - lack of staining, 1 - weak, 2 - strong) of EGFR (A-D), APOBEC3B $(\mathrm{E}-\mathrm{H}), \mathrm{mTOR}(\mathrm{I}-\mathrm{L}), \mathrm{PTEN}(\mathrm{M}-\mathrm{O})$ and $\mathrm{pPTEN}(\mathrm{P}-\mathrm{R})$ is presented on the picture.

EGFR - epidermal growth factor receptor; APOBEC $3 B$ - apolipoprotein B mRNA editing enzyme catalytic subunit $3 B$; mTOR - mammalian target of rapamycin; PTEN - phosphatase and tensin homologue deleted on chromosome ten

3CA_760_mu TaqMan Mutation Detection Assay (Assay ID: Hs00000822_mu) allowing for p. E542K (c.1624G > A) detection and (4) PIK3CA_rf TaqMan Mutation Detection Reference Assay (Assay ID: $\mathrm{Hs} 00001025$ rf) allowing for detection of PIK3CA conservative fragment, which serves as a DNA quality control (indicating whether isolated DNA is of relevant quality to be amplified).

The amplification was carried out in $20 \mu \mathrm{l}$ reaction mixture containing 20 ng DNA, 2 ul of one of TaqMan Mutation Detection Assays, $10 \mu \mathrm{l}$ of TaqMan Genotyping Master Mix, $0.4 \mu \mathrm{l}$ of Exogenous IPC Template DNA and $2 \mu \mathrm{l}$ of Exogenous IPC Mix. All reagents were bought from Applied Biosystems (Foster City, CA, USA). The qPCR cycling con- ditions were as follows: initial denaturation at $95^{\circ} \mathrm{C}$ for $10 \mathrm{~min}$, then 5 cycles of $92^{\circ} \mathrm{C}$ for $15 \mathrm{~s}$ and $58^{\circ} \mathrm{C}$ for $1 \mathrm{~min}$, and finally 50 cycles of $92^{\circ} \mathrm{C}$ for $15 \mathrm{~s}$ followed by $60^{\circ} \mathrm{C}$ for $1 \mathrm{~min}$.

Apart from PIK3CA fragment, internal positive control was amplified in each well due to check of eventually PCR inhibition. Each processing plate contained also 2 wells with nuclease-free water instead of DNA (no template controls). On the basis of obtained qPCR signals, the sample was classified as positive or negative (with or without PIK3CA mutation). We were able to determine mutational status for 152 tissues (for 3 patients obtained DNA was of very low quality and there was no alternative FFPE material). 


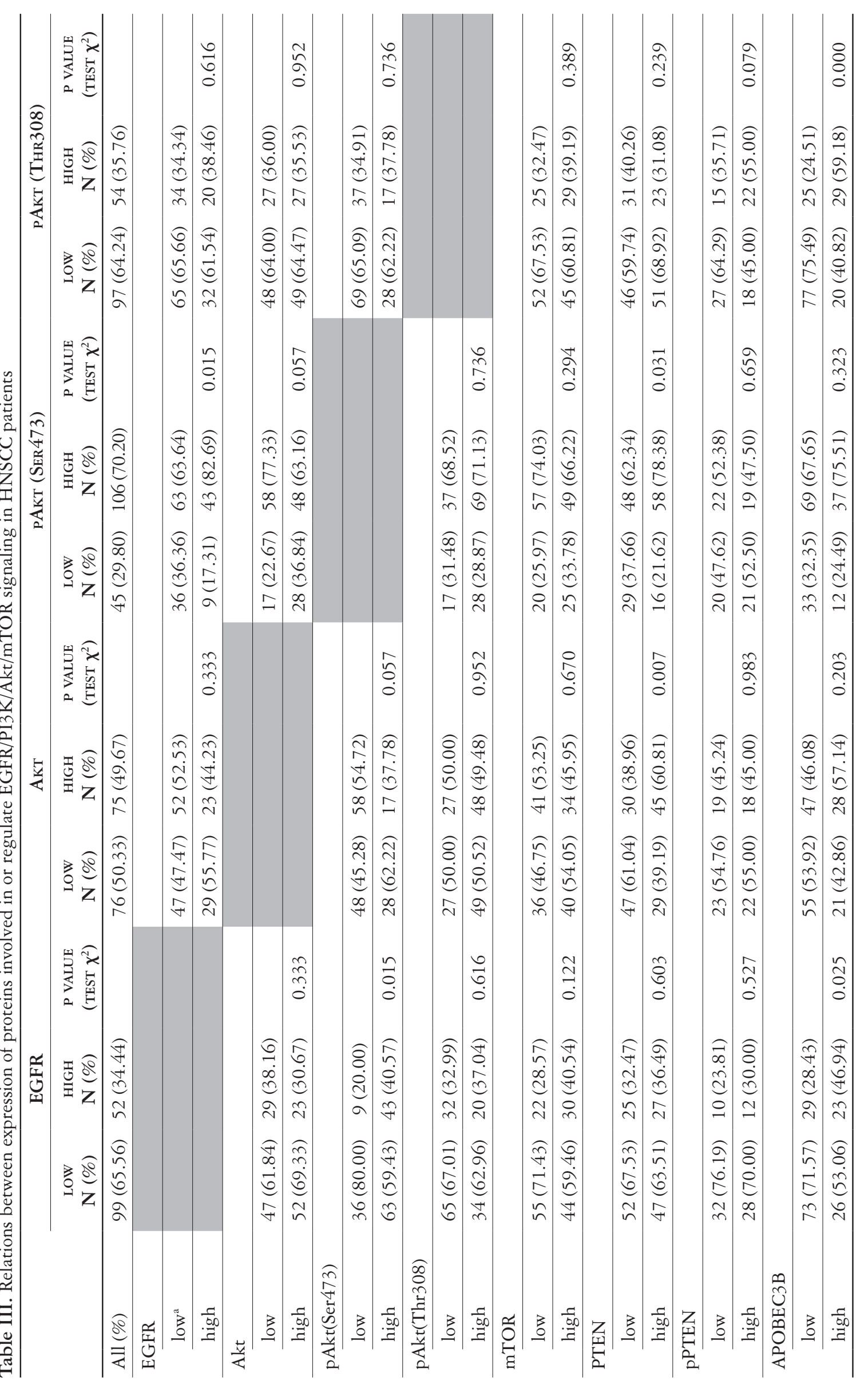




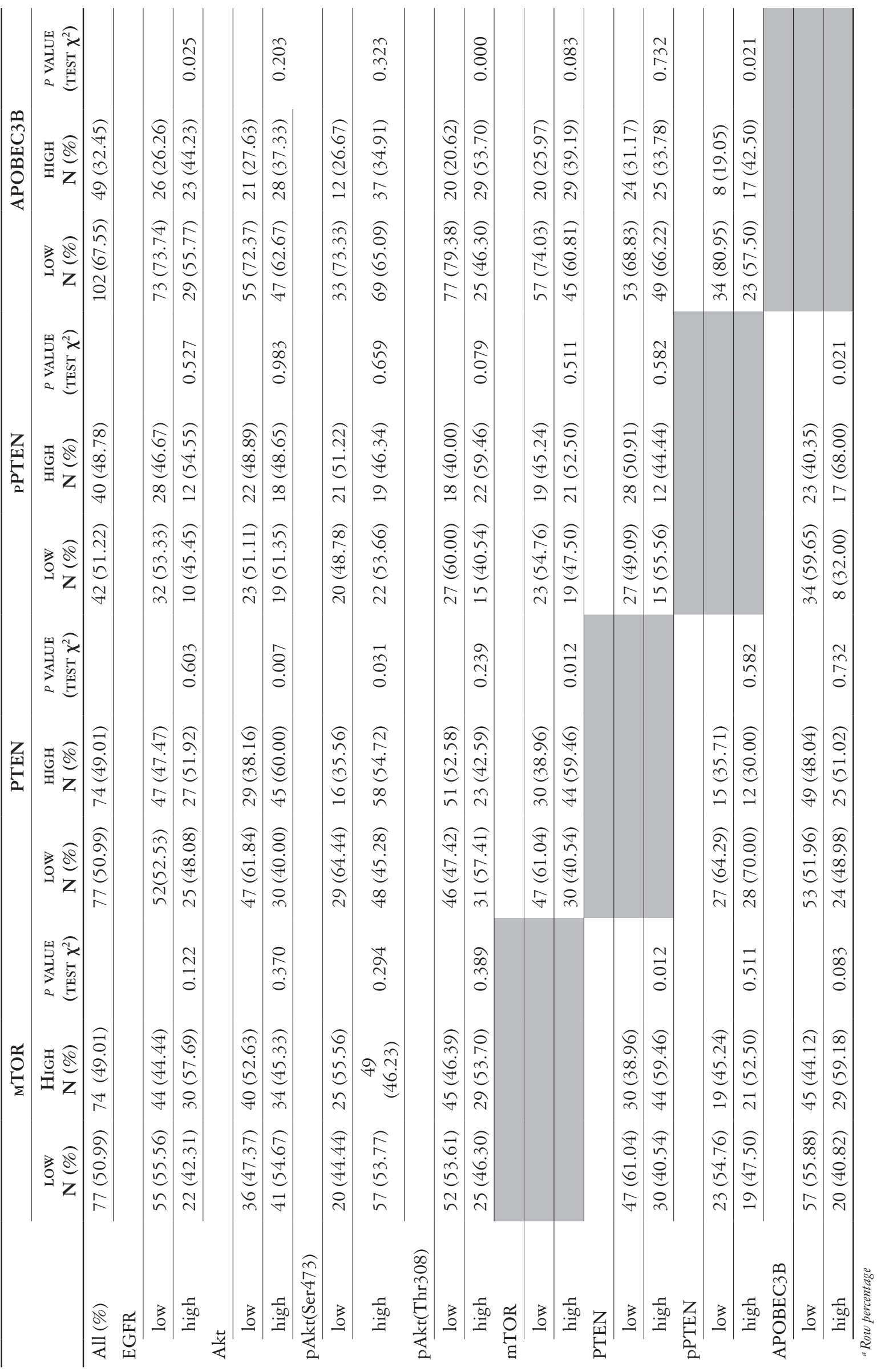




\section{Statistical analyses}

Cut off values were calculated by minimal $\mathrm{p}$ value method and in the cases, where statistically significant differences for some variable was not found, median was used as a cut off point. Descriptive statistics were used for determination of means and medians of continuous variables. Relations between categorical variables were analyzed by Pearson $\chi^{2}$ test. Prognostic potential was analyzed based on 5-year overall survival (OS, time from the end of therapy to death from any cause within 5 years after finishing the treatment) and 5-year disease free survival (DFS, time from the end of therapy to the first documented evidence of recurrent disease i.e. treatment failure, locoregional recurrence or distant metastasis within 5 years after finishing the treatment). Kaplan-Meier method and log-rank test were used for calculation of OS and DFS probabilities. Univariate and multivariate analyses with Cox proportional regression model were carried out for independent prognostic factors selection. All parameters which in univariate analysis were found to statistically significantly influence survival, were included into multivariate analysis. Calculations were performed using Statistica v. 13.3; p value less than 0.05 was considered significant.

\section{Results}

\section{Patients}

Detailed characteristics of HNSCC patients, who had assessed IHC proteins expression, are presented in Table I. Based on simultaneous assessment of immunohistochemical p16 overexpression and HPV16 DNA presence by nested PCR and qPCR, among 151 tumors we found 28 cases with active HPV 16 infection (18.54\%) and 119 (78.81\%) cases with no viral infection (patients infected with other HPV types were excluded from further analyses) [13].

\section{Association between clinicopathological characteristics and expression of EGFR, mTOR, APOBEC3B, PTEN and pPTEN}

In the present study we detected expression of $\mathrm{mTOR}, \mathrm{APOBEC} 3 \mathrm{~B}$ and $\mathrm{pPTEN}$ in all analyzed cases, whereas expressions of PTEN and EGFR were not detected at any level in $23(15.23 \%)$ and $7(4.64 \%)$ tissues, respectively. Clinical and histopathological features in relation to proteins expressions are presented in Table I. Relations between clinicopathological characteristics and expressions of Akt, pAkt(Ser473) and pAkt(Thr308) have been investigated by us earlier [14].
The presence of active HPV16 infection in tumor was significantly associated only with EGFR expression $(\mathrm{p}=0.002)$. Among HPV16 positive HNSCC significantly more tumors with low EGFR expression were noticed as compared to those with HPV negativity ( $89.29 \%$ vs. $58.82 \%$ ). There was no significant differences identified in the level of mTOR $(\mathrm{p}=0.426)$, APOBEC $3 \mathrm{~B}(\mathrm{p}=0.882)$, PTEN $(\mathrm{p}=0.471)$ and $\mathrm{pPTEN}(\mathrm{p}=0.078)$ between HPV positive and negative tumors.

Apart from HPV16 infection, EGFR expression was found to be significantly associated with keratinization status ( $\mathrm{p}=0.015)$ and grade $(\mathrm{p}=0.026)$. Generally, the higher grade, the less tumors with EGFR overexpression identified. To be more precise, within tumors with grade I, II or III there were $48.94,30.12$ and $19.05 \%$ of cases with high level of EGFR detected, respectively. Moreover, a significantly higher percentage of non-keratinizing tumors had low level of EGFR expression (76.56\%) detected than keratinizing ones $(57.47 \%)$. mTOR, in turn, was related only to gender. Females significantly $(\mathrm{p}=0.001)$ more often had high expression of mTOR identified than males (79.17 vs. $43.31 \%$, respectively). Further, APOBEC3B expression was significantly associated with treatment outcome $(\mathrm{p}=0.016)$ and PTEN expression with Karnofsky performance status of patients and localization of tumor. Patients in a good performance status had significantly more often $(67.19 \%, \mathrm{p}=0.001)$ low PTEN expression detected, contrary to those in a worse condition, who had mostly $(60.92 \%)$ high level of PTEN detected. Moreover, low expression of PTEN was statistically significantly less often $(\mathrm{p}=0.000)$ identified in laryngeal tumors $(27.59 \%)$ as compare to oral, oropharyngeal and hypopharyngeal tumors, in which low PTEN expression was detected in 66.67, 65.08 and $66.67 \%$ of cases, respectively. We did not observed any significant relations between clinicopathological features and pPTEN expression.

\section{Relations between proteins involved in EGFR/ $\mathrm{PI} 3 \mathrm{~K} / \mathrm{Akt} / \mathrm{mTOR}$ signaling}

We also checked for the relations between analyzed proteins, including Akt and its two phosphorylated forms - pAkt(Ser473) and pAkt(Thr308). There were few significant associations identified (Table III). Tumors characterized with high expression of EGFR had significantly more often high expression of pAkt(Ser473) as compare to tumors with low EGFR expression (82.69 vs. 63.64\%, respectively, $\mathrm{p}=0.015)$. Tumors with EGFR overexpression had also significantly less often low expression of APOBEC3B than those with low EGFR expression ( 55.77 vs. $73.74 \%$, respectively, $\mathrm{p}=0.025)$. Additionally, expression of APOBEC $3 \mathrm{~B}$ was significantly correlated with pAkt(Thr308) ( $\mathrm{p}=0.000)$ and pPTEN 
$(\mathrm{p}=0.021)$ levels in cancer tissue. Cancers with high level of APOBEC3B had significantly more often also high level of pAkt(Thr308) and high level of pPTEN (59.18 and $68.00 \%$, respectively), contrary to those with low APOBEC3B expression, which had more often low pAkt(Thr308) and low pPTEN signals detected (75.49 and $59.65 \%$, respectively).

Moreover, we observed significant relation between PTEN and Akt ( $\mathrm{p}=0.007)$, pAkt(Ser473) $(\mathrm{p}=0.031)$ and mTOR $(\mathrm{p}=0.012)$ immunohistochemical expressions. Tumors with low PTEN signal had more often low Akt (61.04\%) and low mTOR (61.04\%) expressions, as compare to tumors with high level of PTEN expression, which had mostly high levels of Akt and mTOR (60.81 and 59.46\%, respectively). Unexpectedly, within cancers with low PTEN expression the percentage of tumors highly expressed pAkt(Ser473) was lower than among tumors with high PTEN expression (62.34 vs. 78.38\%, respectively).

We did not found any other relations between analyzed proteins (Table III).

\section{PIK3CA mutations}

We analyzed presence of the most frequent mutations within PIK3CA gene - p.E542K and p.E545K in exon 9 (helical domain) and p.H1047R in exon 20 (kinase domain). We identified 5 mutated tissues in total $(3.29 \%)$. In the HPV16 positive subgroup point mutations only within PIK3CA helical domain were detected. To be more detailed, we identified c. $1633 \mathrm{G}>\mathrm{A}$ (p.E545K) mutation in one case and c.1624G > A (p.E542K) mutation in another one. Hence, the percentage of HPV16 positive tumors with changed PIK3CA sequence was $7.14 \%$. On the other hand, in HPV negative subgroup the frequency was lower (2.5\%). We identified 2 tumors carrying c. $3140 \mathrm{~A}>\mathrm{G}$ (p.H1047R) mutation within kinase domain and 1 carrying c.1633G $>$ A (p.E545K) mutation within helical domain of $P I K 3 C A$ gene. However, the difference in mutational rate between HPV 16 positive and HPV negative HNSCCs did not reach statistical significance.

\section{Survival analyses}

Survival analyses were performed in a group of 151 patients for EGFR, Akt, pAkt(Ser473), pAkt(Thr308), mTOR, APOBEC3B and PTEN (4 tumors with material not sufficient for obtaining IHC data were excluded) and in the case of pPTEN in the subgroup of 82 patients. The mutational status of PIK3CA gene could not be included into survival analyses because of low number of positive cases. The results of univariate analysis are presented in Table IV. The analysis has shown that patients with high EGFR expression had 1.7 times higher probability of death $(\mathrm{p}=0.027)$ and 2.1 times of higher risk of cancer progression (recurrence, developing

Table IV. Univariate Cox proportional hazard model for 5-year overall and disease free survivals of HNSCC patients

\begin{tabular}{|c|c|c|c|c|c|c|c|c|}
\hline & \multicolumn{4}{|c|}{ 5-YEAR OVERALL SURVIVAL } & \multicolumn{4}{|c|}{ 5-YEAR DISEASE FREE SURVIVAL } \\
\hline & $\begin{array}{c}\text { ALIVE/ALL } \\
\text { PATIENTS } \\
(\%)^{\mathrm{A}}\end{array}$ & $\mathrm{HR}$ & $95 \% \mathrm{CI}$ & P VALUE & $\begin{array}{c}\text { Alive/ALL } \\
\text { PATIENTS } \\
(\%)^{\mathrm{A}}\end{array}$ & HR & $95 \% \mathrm{CI}$ & P VALUE \\
\hline \multicolumn{9}{|l|}{ EGFR } \\
\hline Low & $55 / 99(55.56)$ & 1.000 & & & 73/99 (73.74) & 1.000 & & \\
\hline High & $18 / 52(34.62)$ & 1.658 & $1.059-2.595$ & 0.027 & $26 / 52(50.00)$ & 2.142 & $1.242-3.694$ & 0.005 \\
\hline \multicolumn{9}{|l|}{ mTOR } \\
\hline Low & $38 / 77(49.35)$ & 1.132 & $0.726-1.766$ & 0.581 & $50 / 77(64.94)$ & 1.200 & $0.693-2.069$ & 0.506 \\
\hline High & $35 / 74(47.30)$ & 1.000 & & & $49 / 74(66.22)$ & 1.000 & & \\
\hline \multicolumn{9}{|l|}{ PTEN } \\
\hline Low & $40 / 77(51.95)$ & 1.000 & & & $53 / 77(68.83)$ & 1.000 & & \\
\hline High & $33 / 74(44.59)$ & 1.150 & $0.737-1.793$ & 0.535 & $46 / 74(62.16)$ & 1.208 & $0.700-2.084$ & 0.492 \\
\hline \multicolumn{9}{|l|}{ pPTEN } \\
\hline Low & $24 / 42(57.14)$ & 1.093 & $0.563-2.123$ & 0.790 & $32 / 42(76.19)$ & 1.000 & & \\
\hline High & $23 / 40(57.50)$ & 1.000 & & & $25 / 40(62.50)$ & 1.510 & $0.677-3.364$ & 0.306 \\
\hline \multicolumn{9}{|c|}{ АРОВЕСЗВ } \\
\hline Low & $53 / 102(51.96)$ & 1.000 & & & $74 / 102(72.55)$ & 1.000 & & \\
\hline High & $20 / 49(40.82)$ & 1.357 & $0.856-2.151$ & 0.196 & $25 / 49(51.02)$ & 2.026 & $1.173-3.499$ & 0.011 \\
\hline
\end{tabular}


Table V. Multivariate Cox proportional hazard model in HNSCC patients

\begin{tabular}{|c|c|c|c|}
\hline & HR & $95 \% \mathrm{CI}$ & p value ${ }^{a}$ \\
\hline \multicolumn{4}{|c|}{ 5-YEAR OVERALL SURVIVAL } \\
\hline \multicolumn{4}{|l|}{ Gender } \\
\hline Male & 2.530 & $1.013-6.315$ & 0.047 \\
\hline Female & 1.000 & & \\
\hline \multicolumn{4}{|l|}{ T stage } \\
\hline $1+2$ & 1.000 & & \\
\hline $3+4$ & 2.219 & $1.062-4.635$ & 0.034 \\
\hline \multicolumn{4}{|c|}{ pAkt(Thr308) expression } \\
\hline Low & 1.000 & & \\
\hline High & 1.843 & $1.165-2.915$ & 0.009 \\
\hline \multicolumn{4}{|c|}{ HPV16 active infection } \\
\hline Present & 1.000 & & \\
\hline Absent & 3.765 & $1.510-9.388$ & 0.004 \\
\hline \multicolumn{4}{|c|}{ 5-YEAR DISEASE FREE SURVIVAL } \\
\hline \multicolumn{4}{|l|}{ T stage } \\
\hline $1+2$ & 1.000 & & \\
\hline $3+4$ & 3.060 & $1.102-8.498$ & 0.032 \\
\hline \multicolumn{4}{|c|}{ HPV16 active infection } \\
\hline Present & 1.000 & & \\
\hline Absent & 7.276 & $1.767-29.970$ & 0.006 \\
\hline \multicolumn{4}{|c|}{ pAkt(Thr308) expression } \\
\hline Low & 1.000 & & \\
\hline High & 2.245 & $1.293-3.896$ & 0.004 \\
\hline
\end{tabular}

metastasis or treatment failure, $\mathrm{p}=0.005)$ than patients with low level of EGFR expression. Additionally, a statistically significant impact of APOBEC3B expression on 5-year DFS was demonstrated $(\mathrm{p}=0.011)$. Patients having tumors with high APOBEC3B expression detected, had over 2 times higher risk of cancer progression than those with lower level of this protein detected in cancer tissue. A significant impact of $\mathrm{pAkt}(\operatorname{Ser} 473)$ and $\mathrm{pAk}$ $\mathrm{t}(\mathrm{Thr} 308)$ but no total Akt level on OS and DFS in the same group of patients have been demonstrated by us earlier [14]. PTEN, pPTEN and mTOR levels, in turn, did not influenced survival. Clinical and histopathological features significantly affected OS and DFS were also identified by us earlier [13]. For 5-year OS gender, performance status (in Karnofsky scale), the level of smoking, $\mathrm{T}$ and $\mathrm{N}$ stages as well as the presence of active HPV16 infection were found statistically significant. For 5-year DFS, in turn, significant were gender, age, levels of smok- ing and drinking, T stage, grade and active HPV16 infection.

Due to finding independent prognostic factors in the analyzed group of patients, multivariate analysis was performed. All variables exhibiting a significant impact on OS and DFS in univariate analysis were included into multivariate analysis, which results are presented in Table V. It revealed that independent favorable prognostic factors for OS were: (1) female gender $(\mathrm{p}=0.047$, men had over 2.5 times higher risk of death within the 5 years from the end of the treatment), (2) lower $\mathrm{T}$ stage ( $\mathrm{p}=0.034$, patients having tumors in higher $\mathrm{T}$ stage had almost 2.3 times higher risk of death), (3) low expression of pAkt(Thr308) ( $\mathrm{p}=0.009$, patients with tumors expressing pAkt(Thr308) at high level had 1.8 times higher risk of death) and (4) active HPV16 infection presence $(\mathrm{p}=0.004$, patients with no active HPV infection had about 3.8 times higher risk of death). For DFS, in turn, independent prognostic factors were: (1) T stage ( $p=0.032$, patients with tumors in higher $\mathrm{T}$ stage had 3 times higher risk of cancer progression within 5 years after treatment), (2) pAkt(Thr308) expression $(\mathrm{p}=0.004$, patients with tumors expressing pAkt(Thr308) at high level had over 2.2 times higher risk of cancer progression) and ( 3 ) active HPV16 infection $(p=0.006$, patients with no active HPV infection had almost 7.3 times higher risk of cancer progression).

We also analyzed an impact of expression of proteins involved in EGFR/PI3K/Akt/mTOR pathway on survival separately for HPV16 positive and HPV negative patients (Fig. 2). In the subgroup of patients without active viral infection, statistically significant differences in DFS were found between patients having tumors with high and low APOBEC3B $(\mathrm{p}=0.039)$ and Akt ( $\mathrm{p}=0.049)$ expression levels. Additionally, a significant influence on both OS $(\mathrm{p}=0.011)$ and DFS $(\mathrm{p}=0.005)$ for HPV negative patients with different pAkt(Thr308) status was demonstrated. Interestingly, although there was no significant survival improvement in relation to total Akt expression in the whole HNSCC patients group, a favorable impact of low Akt expression level on DFS was demonstrated for subgroup without HPV active infection detected.

On the other hand, in the subgroup of HPV16 positive patients only statistically significant $(p=0.039)$ differences in DFS were found between patients with different APOBEC3B level of expression (patients having tumors with low APOBEC3B expression detected survived longer). Expression of other analyzed proteins have not significant impact on OS and DFS in HPV16 positive patients. However it is worth to emphasize that for some proteins (including pAkt(Thr308)) similar trends as in HPV negative cancers were observed (data not 

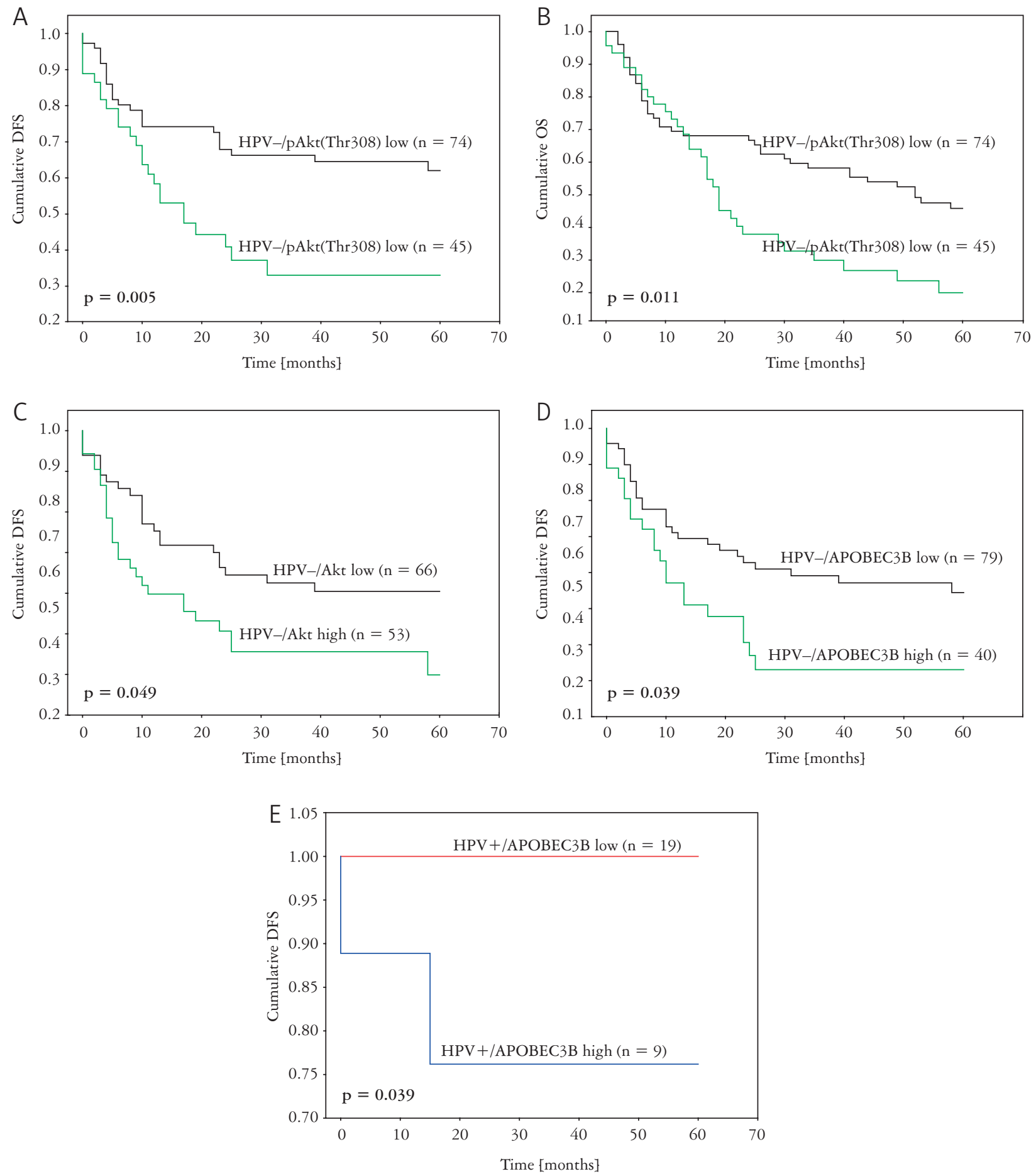

Fig. 2. Cumulative OS and DFS curves of HNSCC patients. Curves for HPV negative HNSCC patients classified by expression of (A and B) pAkt(Thr308), (C) Akt and (D) APOBEC3B as as well as for HPV16 positive patients classified by (E) APOBEC3B status are presented on the graph. Only statistically significant relations have been shown. HPV16 active infection was assessed on the basis of simultaneous HPV16 DNA presence and p16 overexpression in tumor tissue. Protein expressions were assessed immunohistochemically.

HNSCC - head and neck squamous cell carcinoma; OS - overall survival; DFS - disease free survival; HPV - buman papillomavirus; Akt - protein kinase B; $A P O B E C 3 B$ - apolipoprotein $B m R N A$ editing enzyme catalytic subunit $3 B$

shown). Hence, there is a high probability that lack of statistically significance in their cases was the effect of too low number of HPV16 positive cases.
Therefore, increasing the number of patients enrolled to the study would probably verify whether emerging trends are real. 


\section{Discussion}

Activation of EGFR/PI3K/Akt/mTOR pathway occurs in many cancers, including HNSCC. Still little is known about its functioning according to HPV status. Understanding the changes in EGFR/PI3K/ Akt/mTOR pathway related to $\mathrm{HPV}$ infection are extremely important, as they may represent new opportunities for therapeutic intervention. All the more so, because nowadays many inhibitors targeting this pathway are tested in preclinical and clinical studies $[15,16]$.

\section{EGFR}

In the present study we have found EGFR overexpression in $34.3 \%$ of HNSCC. This percentage is similar to those noted by Numico et al. [17] and Owusu-Afriyie et al. [18]. These authors, in the groups of 149 patients with HNSCC and 154 patients with non-oropharyngeal HNSCC, respectively, have demonstrated EGFR overexpression in $35.0 \%$ and $29.4 \%$ cancers. However, in the other papers concerning HNSCC, the percentage of EGFR positivity was significantly higher, in the range from $45.2 \%$ [19] to $100 \%$ [20]. One of the reason of above-mentioned discrepancies may be related to different immunoscores and cut off points used for distinguish tumors with EGFR overexpression or its lack. Some authors decided to divide analyzed tumors based on EGFR positivity/negativity $[17,21]$, but others on EGFR overexpression/lack of overexpression [18, 22, 23]. Moreover, there are different cut-off points applied to distinguish EGFR overexpression/lack of overexpression. Taberna et al. [23], Murray et al. [24] and Bernardes et al. [25] assumed as a cut off point week or moderate staining or strong staining in more than $10 \%$ of tumor cells. In turn, Owusu-Afriyie et al. [18] applied immunoreactive score (multiplication of intensity of staining and percentage of positive staining cells, range from 0 to 12) with cut off point at the level of 4, and Atkins et al. [26] categorized analyzed tumors according EGFR expression into four classes: no, weak, moderate and strong staining.

There are also conflicting results concerning correlation between EGFR expression and epidemiological, clinical and histopathological features. Some authors, similar to us, did not find significant relation between EGFR expression and patient's age, smoking and alcohol abuse $[17,27,28]$, TN stages $[17$, 21, 22], grade [21] or degree of keratinization [19]. However, some researchers reported contrary results, demonstrating significant higher percentage of tumors with EGFR overexpression in older patients [20], smokers [21, 29] and among T3-T4 cancers [19] as compared, respectively, to younger patients, non-smokers or tumors with lower $\mathrm{T}$ stage. Other authors found significant correlation between EGFR expression and grade, i.e. higher number of tumors with EGFR overexpression in grade $3[17,28]$. These opposite results can be partly explain by the differentiation in EGFR expression in specified localization of HNSCC. Srivastava et al. [27] noticed higher expression in oral cancers than in other localization of HNSCC, however we did not confirm such relation in the present study.

It should be also pointed out, that in the present study we have shown significant relation between HPV infection and EGFR expression. Tumors with HPV16 active infection were characterized by significantly lower EGFR expression. This finding is in agreement with results presented by other authors. Taberna et al. [23], in the group of 788 oropharyngeal cancers, reported significantly lower percentage of tumors with EGFR expression among HPV positive cases $(37.7 \%)$ than in those with HPV negativity (70.8\%). This type of relation was also confirmed by Sivarajah et al. [30]. They demonstrated inverse relationship between EGFR expression and CDKN2A levels (gene for p16) in HPV positive and negative HNSCC cell lines. Additionally, in the present study in the subgroup of HPV16 positive patients, EGFR expression did not influence OS and DFS. These all results suggest that the effectiveness of cetuximab (used as a substitute for cisplatin in concomitant chemoradiotherapy in one of de-escalation strategies in the treatment of patients with HPV positive oropharyngeal SCC) may be questioned, because the expression of EGFR in HPV positive HNSCC is lower than in HPV negative ones. This suggestion is confirmed by the results of two phase III completed trials (RTOG 1016 and De-ESCALaTE), in which HPV positive oropharyngeal SCC patients were randomly assigned to receive radiotherapy with concurrent cetuximab or cisplatin. These studies showed significant better OS and locoregional control in the arm with cisplatin $[31,32]$. On the other hand, we found that HNSCC patients with EGFR overexpression have poorer prognosis as compared to those with no/ low EGFR expression, irrespective of HPV status. Similarly, other authors reported EGFR overexpression as negative prognostic factor in HNSCC patients treated with radiotherapy or chemoradiotherapy $[23$, $30,33]$. Taking into account all above mentioned results, it may be that cetuximab can be more effective in HPV negative patients.

On the other hand, in the present study we have found significant correlation between high expression of EGFR and high expression of pAkt(Ser473). High expression of $\mathrm{pAkt}(\operatorname{Ser} 473)$ is associated with active form of Akt enzyme and had in our earlier study negative influence patients survival [14]. Therefore, summarized this part of discussion, it should be pointed out that the question about prognostic value of EGFR expression is still open and the further 
studies should be focus on validation of immunohistochemistry staining and scoring as well as on explaining the differences in EGFR expression between HPV positive and negative cancers.

\section{PIK3CA gene and APOBEC3B}

PIK3CA is one of the most commonly mutated and extensively studied oncogenes in various types of human cancer, including HNSCC. In this study we analyzed presence of the most frequent mutations within PIK3CA gene - p.E542K and p.E545K within helical domain and p.H1047R within kinase domain, representing $73 \%$ of PIK3CA mutations [34]. Data has shown that the rate of PIK3CA mutations in HNSCC is rather low. Cohen et al. [35] found PIK3CA alterations in $10.8 \%$ of oral SCC, Kozaki et al. [36] in $7 \%$ of oral cancer samples and Murugan et al. [37] in 5\% of tumor samples from different sites of head and neck region. Also in our study low number of tumors harboring PIK3CA mutations (3.29\%) was identified.

Despite the low prevalence of PIK3CA mutations in HNSCC, it is worth to emphasize, that the significant differences in their frequency and distribution have been observed between HPV positive and negative tumors. Interestingly, PIK3CA mutations in HPV positive HNSCCs are concentrated in helical domain, whereas HPV negative tumors have mutations throughout the entire gene [34]. Stransky et al. [38] found that 27\% of HPV positive and 5\% of HPV negative samples harbored PIK3CA mutations. Nichols et al. [39] observed similar relations. They detected significantly lower frequency of activating PIK3CA mutations in HPV negative $(10 \%$, 3 cases at codon 1047 and 1 at codon 542) as compared to HPV positive (28\%, 7 cases with mutations at codon 542, 5 at codon 545, and 1 at codon 1047) tumors. Moreover, they noticed that HPV positive patients were more likely to harbor mutations at codons 542 and 545 (12 of 13 mutations), while 3 of 4 mutations in HPV negative tumors occurred at codon 1047. In our study quite lower frequency of mutated HPV negative (2.5\%) and HPV16 positive $(7.14 \%)$ samples were detected, however the distribution of genetic changes was similar to this identified by Nichols et al. In HPV negative tumors we found mutations in both kinase and helical domains ( 2 tumors with p.H1047R and 1 with p.E545K mutations) and in HPV16 positive ones alterations only within PIK3CA helical domain (p.E545K mutation in one case and p.E542K mutation in another one) were observed.

Taking together, our and presented in the literature data have shown that the PIK3CA gene exhibited a higher number of helical domain mutations in the HPV positive population, however these results need to be verify in larger group of patients. This ob- servation may suggest that it exists some HPV-specific mechanism leading to genetic instability in PIK3CA gene. There are some studies suggesting that the higher rate of specific PIK3CA mutations in HPV positive tumors could be the effect of APOBEC $3 \mathrm{~B}$ action.

APOBEC 3B is a member of human APOBEC 3 family. Its fundamental biochemical role is DNA cytosine to uracil deamination activity [40] and the main biological function is to protect human cells against retroviruses and retrotransposons [41]. The APOBEC3B recognizes TCW motifs in DNA (5'-TCA and 5'-TCT trinucleotide motifs) causing $C>T$ and $C>G$ changes [42]. It has been shown that the APOBEC 3B mutation signature is specifically enriched in different types of tumors, including head and neck [43]. It is important to notice that mutations within helical domain of PIK3CA (p.E542K and p.E545K - they are TCW type) may, then, be caused by APOBEC 3B and p.H1047R within kinase domain not, because it is not TCW type.

Henderson et al. [9] assessed hot spot mutations in PIK3CA gene. They found that APOBEC3B expression was elevated in HPV positive HNSCC. Additionally, they observed significantly more TCW mutations of PIK3CA (p.E542K and p.E545K) in HPV positive tumors comparing to HPV negative ones. In HPV positive HNSCC mutations of PIK$3 C A$ were almost exclusively of the TCW type, with the majority occurring at the helical domain, while no p.H1047R mutation was seen. In HPV negative HNSCC, in turn, only 22 of 48 PIK3CA mutations were TCW and they detected 8 p.H1047R mutations. They observed that in tumors with low APOBEC activity, PIK3CA was equally likely to be mutated at the kinase and helical domains. Summing up, they demonstrated that in HPV-induced HNSCC APOBEC $3 B$ activity is responsible for the generation of helical domain hot spot mutations (p.E542K, p.E545K) in the PIK3CA gene. Our results are in agreement with mentioned above. Although we have not observed any significant differences in the level of APOBEC3B between HPV positive and negative tumors, we found high APOBEC $3 \mathrm{~B}$ expression in 2 HPV16 positive tumors with PIK3CA mutations (both in helical domain), whereas $3 \mathrm{HPV}$ negative tumors harboring PIK3CA mutations had low level of APOBEC3B expression.

Vieira et al. [10] also demonstrated the higher APOBEC3B levels in head and neck HPV positive than in HPV negative cancers. They demonstrated that high-risk (but not low-risk) E6 is sufficient for the induction of APOBEC $3 \mathrm{~B}$ expression in keratinocytes and that continuous expression of $\mathrm{E} 6$ is required to maintain higher APOBEC3B levels in HPV positive cancer cell lines. Mori et al. $[44,45]$ have found that $\mathrm{E} 6$ induces upregulation of $\mathrm{APOBEC} 3 \mathrm{~B}$ through increased levels of TEAD family. 
In analyzed group of patients higher expression of APOBEC 3B was observed significantly more often among patients, who revealed distant metastases and among people with treatment failure, contrary to healthy patients at the last follow-up, but also people with local recurrences or died from non-cancer reasons. Moreover, we demonstrated a statistically significant impact of APOBEC3B expression on 5-year DFS. The statistical significance in DFS was maintained also when we performed separate analyses in the subgroup of HPV16 positive and HPV negative patients. Interestingly, APOBEC3B was the only protein in our analysis, which turned out to influence survival in patients with active viral infection. Tsuboi et al. [46] in breast cancer patients have not found any relation between $\mathrm{APOBEC} 3 \mathrm{~B}$ expression and survival, however in their group high APOBEC $3 \mathrm{~B}$ expression was associated with progression of lymph node metastasis and grade. Taking our and Tsuboi et al. data together, it seems that high expression of APOBEC3B may stimulate some changes leading to progression of cancer disease with revealing metastases and treatment resistance. However, this hypothesis needs to be verify in further studies.

To sum up, PI3K and APOBEC 3 B may represent an important predictive biomarkers and therapeutic targets particularly in HPV positive HNSCC patients. Targeting them with molecular agents may be the mechanism to improve cure rates and decrease treatment toxic effects in this rapidly growing cohort of patients. However, further researches are needed to explain the mechanism of HPV-related APOBEC3B overexpression in HNSCC patients and the potential role of $\mathrm{APOBEC} 3 \mathrm{~B}$ in inducing PIK3CA mutations in their tumors.

\section{mTOR}

mTOR is a key downstream regulator of PIK3 pathway, which regulates cell growth, proliferation and progression of cancer. However, in the present study we have not found significant relations between mTOR expression and HPV16 presence or patients' survival. Similar to us, Kiessling et al. [47] in 184 patients with oropharyngeal SCC did not report significant influence of mTOR expression on survival, however, contrary to us, they found significantly higher incidence of mTOR overexpression among p16 negative tumors. In turn, some authors, in the groups of patients with oropharyngeal cancers [48, 49], have reported opposite results, i.e. inferior survival for patients with tumors overexpressing mTOR. It is difficult to indicate clearly the reasons for the described discrepancies, but one should pay attention to the heterogeneity of the studied groups in terms of size, clinical stage or treatment regimes. It should be also noticed that in above-mentioned reports, HPV infection was assessed by p16 immunoex- pression. This protein is a surrogate marker of HPV presence and its use is related to risk of false positive results obtainment. On the other hand, taking into account the possibility of treatment with mTOR inhibitors, such as everolimus [48] or rapamycin [50] in HNSCC patients, the question about mutual relation between HPV infection and mTOR expression requires further studies.

\section{PTEN and PPTEN}

PTEN is a tumor suppressor gene encodes a lipid and protein phosphatase that is involved in regulation of a variety signaling pathways, including PI $3 \mathrm{~K} / \mathrm{Akt}$ pathway. The main mechanism of tumor suppression by PTEN is the maintenance of cellular PIP-3 at low levels, thus inhibiting the PI3K/Akt pathway [6]. Several phosphorylation sites have been identified in PTEN, such as Ser380, Thr382 and Thr383 [51]. Phosphorylation of PTEN at residues Ser380/ Thr382/383 leads to loss of phosphatase activity and tumor suppressor function [52] We identified $48.8 \%$ of HNSCC tumors with high level of pPTEN. Yang et al. [52] similarly assessed by IHC the phosphorylation of PTEN but throughout the various stages of gastric cancer. They concluded that reduced expression of PTEN and increased PTEN phosphorylation at residues Ser380/Thr382/383 could contribute to gastric carcinogenesis. Probably similar effect might be seen in HNSCC, however further studies are needed to verify this hypothesis.

Generally, PTEN has been found frequently inactivated in various human cancers [53]. A loss/decrease of PTEN expression results in $\mathrm{PI} 3 \mathrm{~K} / \mathrm{Akt} / \mathrm{mTOR}$ pathway activation. The majority of PTEN gene dysfunctions has been attributed to mutations, loss of heterozygosity $(\mathrm{LOH})$ or epigenetic silencing, but the data on their frequency are inconsistent $[54,55$, 56]. Not all researchers checked for genetic changes. Because majority of genetic alterations cause loss or decrease of PTEN expression, also this parameter is widely analyzed. Loss of PTEN expression have been demonstrated, then, in 23 to $61 \%$ of HNSCC samples $[47,57,58]$. In analyzed by us group of patients PTEN expression was not detected at any level only in $15.3 \%$ tissues.

We analyzed protein expressions in relation to HPV status. We have not found any significant differences in the level of PTEN nor pPTEN between HPV positive and negative tumors. However, there are some studies where significance has been demonstrated. Chun et al. [12] assessed the expression of PTEN (evaluated by IHC) in 65 tonsillar SCC tumors. Negative PTEN expression was significantly more frequently observed in HPV negative than positive cancers (57\% vs. $27 \%$, respectively). In total, PTEN expression was lost in $47 \%$ of analyzed tumors, what is much higher than we identi- 
fied (15.3\%). On the other hand, when we analyze tumors with low (not loss of) PTEN expression, we found higher frequency $(51.0 \%)$ than Squarize et al. [59], who identified 31.2\% of HNSCCs exhibiting reduced PTEN expression.

There could be many reasons of mentioned frequency discrepancies. There are differences in experimental groups and experiments designing, as well as methodological differences concerning IHC staining procedure and scoring systems or cut off points used for qualification of sample as positive or negative.

In our study PTEN and pPTEN expressions have not influenced OS and DFS in the whole group, neither in HPV16 positive and HPV negative HNSCC patients, when analyzed separately. Similar results were obtained by Kiesling et al. [47]. In their group of patients, PTEN also did not have significant impact on survival in patients with HPV associated oropharyngeal SCC. However, the lack/decreased PTEN expression may possibly results in more aggressive tumors and poor prognosis, due to loss of its suppressor function and activation of $\mathrm{PI} 3 \mathrm{~K} / \mathrm{Akt} / \mathrm{mTOR}$ signaling. Indeed, there are many studies, in which PTEN association with survival in HNSCC patients have been proved. Lee et al. [57] showed that absence of PTEN expression was independent prognostic indicator for clinical outcome in tongue squamous cell carcinoma patients and da Costa et al. [60] demonstrated the negative impact of low PTEN expression on PFS and OS in patients treated with chemotherapy plus cetuximab. Finally, Snietura et al. [61] have demonstrated that low PTEN expression was associated not only to unfavorable LRC, but also to lack of improvement in LRC from accelerated fractionation. PTEN expression turned out to be one of four parameters significantly and independently related to LRC.

Many studies on head and neck cancer patients also analyzed the association between PTEN expression and clinical and histopathological parameters, but the data are again not consistent. Some researches revealed the significant association between no/low PTEN expression and $\mathrm{T}$ stage, $\mathrm{N}$ stage and/or grade $[58,62]$. However, there are studies where such relations were not found [61, 63], similarly to us. On the other hand, in our study expression of PTEN was significantly associated with cancer site. In laryngeal tumors we observed significantly less often decreased PTEN expression (27.59\%) as compare to oral, oropharyngeal and hypopharyngeal tumors, in which low PTEN level was detected in 66.67, 65.08 and $66.67 \%$ of cases, respectively. Snietura et al. [61] observed opposite results (slightly more laryngeal than oral cavity/oropharyngeal tumors had low expression of PTEN: 62.2 vs. 57.3\%, respectively) and Ahmed et al. [62] highly significant downregulation of PTEN demonstrated in tumors of the oral cavity compared with laryngeal and pharyngeal cancer tissues. All mentioned variances might result again from different number of HNSCC cases enrolled in the particular studies as well as different criteria of qualification to the study and some methodological discrepancies.

Apart from correlations with clinicopathological features, we checked for associations between proteins involved in EGFR/PI3K/Akt/mTOR pathway. Some studies have shown expected significant correlation between pAkt(Ser473) positivity and loss of PTEN expression [58]. Surprisingly, we presented opposite results. In analyzed by us group of HNSCC patients, within cancers with low PTEN expression the percentage of tumors highly expressed pAkt(Ser473) was significantly lower than among tumors with high PTEN expression (62.34 vs. $78.38 \%$, respectively). Similar observations have been already published. Turk et al. [63] detected a relationship between high pAkt1 staining and normal (not decreased) expression of PTEN. It should be take into account the possibility that in some tumors PTEN does not function properly due to point mutations or other mechanisms, which do not cause large deletions or protein loss (therefore it is not possible to catch such cases by IHC - PTEN staining is seen but the protein does not work). Additionally, Akt activation may be stimulated by proteins not affected PTEN expression. In the literature an association with EGFR has been also analyzed. We have not found any relations between PTEN and EGFR status, what is in agreement with othe authors [60, 61].

The published data suggest that PTEN and pPTEN may be a candidates for valuable prognostic and/or predictive biomarkers in HNSCC patients, however additional investigation has to be performed, since prior studies have yielded highly discordant results.

\section{Conclusions}

Understanding the changes in EGFR/PI3K/Akt/ mTOR pathway related to HPV infection are extremely important because may represent new opportunities for therapeutic intervention. To the best of our knowledge, this is the first report of such comprehensive analysis of EGFR/PI3K/AKT/mTOR pathway components performed in HPV16 positive and HPV negative HNSCC patients. In the present study we analyzed the frequency of PIK3CA mutations and expression of 8 proteins involved or regulate EGFR/PI3K/Akt/mTOR signaling as well as their prognostic potential. Obtained results suggest the existence of significant differences in EGFR/ $\mathrm{PI} 3 \mathrm{~K} / \mathrm{Akt} / \mathrm{mTOR}$ pathway functioning between HNSCC patients with different HPV status. Further understanding the molecular differences in functioning of EGFR/PI3K/Akt/mTOR pathway in HPV-de- 
pendent and non-dependent tumors (especially concerning EGFR, Akt and pAkt expressions) may help in individualization of anticancer therapy and in the consequence - improve results of treatment and patients survival.

The authors declare no conflict of interest.

The study was financed by National Science Centre, Poland project No. 2016/21/N/NZ5/00227.

\section{References}

1. Wang H, Sun R, Lin H, et al. P16INK4A as a surrogate biomarker for human papillomavirus-associated oropharyngeal carcinoma: Consideration of some aspects. Cancer Sci 2013; 104: 1553-1559.

2. Licitra L, Perrone F, Bossi P, et al. High-risk human papillomavirus affects prognosis in patients with surgically treated oropharyngeal squamous cell carcinoma. J Clin Oncol 2006; 24: 5630-5636.

3. Lassen P, Eriksen JG, Hamilton-Dutoit S, et al. Effect of HPV-associated p16INK4A expression on response to radiotherapy and survival in squamous cell carcinoma of the head and neck. J Clin Oncol 2009; 27: 1992-1998.

4. Fakhry C, Westra WH, Li S, et al. Improved survival of patients with human papillomavirus-positive head and neck squamous cell carcinoma in a prospective clinical trial. J Natl Cancer Inst 2008; 100: 261-269.

5. Ghittoni R, Accardi R, Chiocca S, et al. Role of human papillomaviruses in carcinogenesis. Ecancermedicalscience 2015; 9: 526.

6. Dahia PL. PTEN, a unique tumor suppressor gene. Endocr Relat Cancer 2000; 7: 115-129.

7. Suh Y, Amelio I, Guerrero Urbano T, et al. Clinical update on cancer: molecular oncology of head and neck cancer. Cell Death Dis 2014; 5: e1018.

8. Nichols AC, Palma DA, Chow W, et al. High frequency of activating PIK3CA mutations in human papillomavirus-positive oropharyngeal cancer. JAMA Otolaryngol Head Neck Surg 2013; 139: 617-622.

9. Henderson S, Chakravarthy A, Su X, et al. APOBEC-mediated cytosine deamination links PIK3CA helical domain mutations to human papillomavirus-driven tumor development. Cell Rep 2014; 7: 1833-1841.

10. Vieira VC, Leonard B, White EA, et al. Human papillomavirus E6 triggers upregulation of the antiviral and cancer genomic DNA deaminase APOBEC3B. mBio 2014; 5: e02234-14.

11. Pollock NI, Wang L, Wallweber G, et al. Increased expression of HER2, HER3, and HER2:HER3 heterodimers in HPV-positive HNSCC using a novel proximity-based assay: Implications for targeted therapies. Clin Cancer Res 2015; 21: 4597-4606.

12. Chun SH, Jung CK, Won HS, et al. Divergence of P53, PTEN, $\mathrm{PI} 3 \mathrm{~K}, \mathrm{Akt}$ and mTOR expression in tonsillar cancer. Head Neck 2015; 37: 636-643.

13. Janecka-Widła A, Mucha-Małecka A, Majchrzyk K, et al. Active HPV infection and its influence on survival in head and neck squamous-cell cancer. J Cancer Res Clin Oncol 2020; 146: 1677-1692.

14. Janecka-Widła A, Majchrzyk K, Mucha-Małecka A, et al. Prognostic potential of Akt, pAkt(Ser473) and pAkt(Thr308) immunoreactivity in relation to HPV prevalence in head and neck squamous cell carcinoma patients. Pathol Res Pract 2021; 229: 153684 .

15. Jung K, Kang H, Mehra R. Targeting phosphoinositide 3-kinase $(\mathrm{PI} 3 \mathrm{~K})$ in head and neck squamous cell carcinoma (HNSCC). Cancers Head Neck 2018; 3: 3.
16. Harsha C, Banik K, Ang HL, et al. Targeting AKT/mTOR in oral cancer: Mechanisms and advances in clinical trials. Int J Mol Sci 2020; 21: 3285.

17. Numico G, Russi EG, Colantonio I, et al. EGFR status and prognosis of patients with locally advanced head and neck cancer treated with chemoradiotherapy. Anticancer Res 2010; 30: 671-676.

18. Owusu-Afriyie O, Owiredu WKBA, Owusu-Danquah K, et al. Expression of immunohistochemical markers in non-oropharyngeal head and neck squamous cell carcinoma in Ghana. PLoS One 2018; 13: e0202790.

19. Hashmi AA, Hussain ZF, Aijaz S, et al. Immunohistochemical expression of epidermal growth factor receptor (EGFR) in South Asian head and neck squamous cell carcinoma: association with various risk factors and clinico-pathologic and prognostic parameters. World J Surg Oncol 2018; 16: 118.

20. Mahendra A, Shreedhar B, Kamboj M, et al. Epidermal growth factor receptor protein: A biological marker for oral precancer and cancer. Journal of Dental Surgery 2014; 2014: 158709.

21. Sarkis SA, Abdullah BH, Abdul Majeed BA, et al. Immunohistochemical expression of epidermal growth factor receptor (EGFR) in oral squamous cell carcinoma in relation to proliferation, apoptosis, angiogenesis and lymphangiogenesis. Head Neck Oncol 2010; 2: 13.

22. Maiti GP, Mondal P, Mukherjee N, et al. Overexpression of EGFR in head and neck squamous cell carcinoma is associated with inactivation of SH3GL2 and CDC25A genes. PLoS One 2013; 8: e63440.

23. Taberna M, Torres M, Alejo M, et al. The use of HPV16-E5, EGFR, and pEGFR as prognostic biomarkers for oropharyngeal cancer patients. Front Oncol 2018; 8: 589.

24. Murray S, Bobos M, Angouridakis N, et al. Screening for EGFR mutations in patients with head and neck cancer treated with gefitinib on a compassionate-use program: A Hellenic Cooperative Oncology Group Study. J Oncol 2010; 2010: 709678.

25. Bernardes VF, Gleber-Netto FO, Sousa SF, et al. EGFR status in oral squamous cell carcinoma: Comparing immunohistochemistry, FISH and CISH detection in a case series study. BMJ Open 2013; 3: e002077.

26. Atkins D, Reiffen K-A, Tegtmeier CL, et al. Immunohistochemical detection of EGFR in paraffin-embedded tumor tissues: Variation in staining intensity due to choice of fixative and storage time of tissue sections. J Histochem Cytochem 2004; 52: 893-901.

27. Srivastava V, Kumar M, Chaturvedi TP, et al. EGFR over-expression in head and neck squamous carcinoma \& its correlation with etiological factors like alcohol and tobacco. World J Pathol 2016; 5: 16-23.

28. Rathore S, Rashmi MV, Singh PK. EGFR scoring in head and neck squamous cell carcinoma and its association with clinicopathological variables. Int J Med Res Rev 2017; 5: 731-739.

29. Belobrov S, Angel C, Wiesenfeld D, et al. Histopathological analysis of oral squamous cell carcinoma in nonsmokers and nondrinkers. Trans Res Oral Oncol 2016; 1: 1-10.

30. Sivarajah S, Kostiuk M, Lindsay C, et al. EGFR as a biomarker of smoking status and survival in oropharyngeal squamous cell carcinoma. J Otolaryngol Head Neck Surg 2019; 48: 1-8.

31. Gillison ML, Trotti AM, Harris J, et al. Radiotherapy plus cetuximab or cisplatin in human papillomavirus-positive oropharyngeal cancer (NRG Oncology RTOG 1016): A randomised, multicentre, non-inferiority trial. Lancet 2019; 393: 40-50.

32. Mehanna H, Robinson M, Hartley A, et al. Radiotherapy plus cisplatin or cetuximab in low-risk human papillomavirus-positive oropharyngeal cancer (De-ESCALaTE HPV): An open-label randomised controlled phase 3 trial. Lancet 2019; 393: 51-60.

33. Wheeler S, Siwak DR, Chai R, et al. Tumor epidermal growth factor receptor and EGFR PY1068 are independent prognostic 
indicators for head and neck squamous cell carcinoma. Clin Cancer Res 2012; 18: 2278-2289.

34. The Cancer Genome Atlas Network. Comprehensive genomic characterization of head and neck squamous cell carcinomas. Nature 2015; 517: 576-582.

35. Cohen Y, Goldenberg-Cohen N, Shalmon B, et al. Mutational analysis of PTEN/PIK3CA/AKT pathway in oral squamous cell carcinoma. Oral Oncol 2011; 47: 946-950.

36. Kozaki K, Imoto I, Pimkhaokham A, et al. PIK3CA mutation is an oncogenic aberration at advanced stages of oral squamous cell carcinoma. Cancer Sci 2006; 97: 1351-1358.

37. Murugan AK, Hong NT, Fukui Y, et al. Oncogenic mutations of the PIK3CA gene in head and neck squamous cell carcinomas. Int J Oncol 2008; 32: 101-111.

38.39. Stransky N, Egloff AM, Tward AD, et al. The mutational landscape of head and neck squamous cell carcinoma. Science 2011; 333: 1157-1160.

40. Nichols AC, Palma DA, Chow W, et al. High frequency of activating PIK3CA mutations in human papillomavirus-positive oropharyngeal cancer. JAMA Otolaryngol Head Neck Surg 2013; 139: 617-622.

41. Harris RS, Dudley JP. APOBECs and virus restriction. Virology 2015 ; 479-480: 131-145.

42. Zou J, Wang C, Ma X, et al. APOBEC3B, a molecular driver of mutagenesis in human cancers. Cell Biosci 2017; 7: 29.

43. Alexandrov, L., Nik-Zainal, S., Wedge, D. et al. Signatures of mutational processes in human cancer. Nature 2013; 500: 415-421.

44. Burns MB, Temiz NA, Harris RS. Evidence for APOBEC3B mutagenesis in multiple human cancers. Nat Genet 2013; 45: 977-983.

45. Mori S, Takeuchi T, Ishii $Y$, et al. Identification of APOBEC3B promoter elements responsible for activation by human papillomavirus type 16 E6. Biochem Biophys Res Commun 2015; 460: 555-560.

46. Mori S, Takeuchi T, Ishii Y, et al. Human papillomavirus 16 E6 upregulates APOBEC3B via the TEAD transcription factor. J Virol 2017; 91: e02413-16

47. Tsuboi, M., Yamane, A., Horiguchi, J. et al. APOBEC3B high expression status is associated with aggressive phenotype in Japanese breast cancers. Breast Cancer 2016; 23: 780-788.

48. Kiessling SY, Broglie MA, Soltermann A, et al. Comparison of PI3K pathway in HPV-associated oropharyngeal cancer with and without tobacco exposure. Laryngoscope Investig Otolaryngol 2018; 3: 283-289.

49. Li SH, Chien CY, Huang WT, et al. Prognostic significance and function of mammalian target of rapamycin in tongue squamous cell carcinoma. Sci Rep 2017; 7: 8178.

50. Kawasaki G, Naruse T, Furukawa K, et al. mTORC1 and mTORC2 expression levels in oral squamous cell carcinoma: An immunohistochemical and clinicopathological study. Anticancer Research Mar 2018; 38: 1623-1628.

51. Molinolo AA, Marsh C, El Dinali M, et al. mTOR as a molecular target in HPV-associated oral and cervical squamous carcinomas. Clin Cancer Res 2012; 18: 2558-2568.

52. Vazquez F, Ramaswamy S, Nakamura N, et al. Phosphorylation of the PTEN tail regulates protein stability and function. Mol Cell Biol 2000; 20: 5010-5018.

53. Yang Z, Xie C, Xu W, et al. Phosphorylation and inactivation of PTEN at residues Ser380/Thr382/383 induced by Helicobacter pylori promotes gastric epithelial cell survival through PI3K/Akt pathway. Oncotarget 2015; 6: 31916-31926.

54. Milella M, Falcone I, Conciatori F, et al. PTEN: Multiple functions in human malignant tumors. Front Oncol 2015; 5: 24.

55. Poetsch M, Lorenz G, Kleist B. Detection of new PTEN/ MMAC1 mutations in head and neck squamous cell carcinomas with loss of chromosome 10. Cancer Genet Cytogenet 2002; 132: 20-24.
56. Lui VW, Hedberg ML, Li H, et al. Frequent mutation of the PI3K pathway in head and neck cancer defines predictive biomarkers. Cancer Discov 2013; 3: 761-769.

57. Chung $\mathrm{CH}$, Guthrie VB, Masica DL, et al. Genomic alterations in head and neck squamous cell carcinoma determined by cancer gene-targeted sequencing. Ann Oncol 2015; 26: 1216-1223.

58. Lee JI, Soria JC, Hassan KA, et al. Loss of PTEN expression as a prognostic marker for tongue cancer. Arch Otolaryngol Head Neck Surg 2001; 127: 1441-1445.

59. Alyasiri NS, Mehdi SJ, Alam MS, et al. PTEN-mediated AKT activation contributes to the reduced apoptosis among Indian oral squamous cell carcinoma patients. J Cancer Res Clin Oncol 2012; 138: 103-109

60. Squarize CH, Castilho RM, Abrahao AC, et al. PTEN deficiency contributes to the development and progression of head and neck cancer. Neoplasia 2013; 15: 461-471.

61. da Costa AA, D’Almeida Costa F, Ribeiro AR, et al. Low PTEN expression is associated with worse overall survival in head and neck squamous cell carcinoma patients treated with chemotherapy and cetuximab. Int J Clin Oncol 2015; 20: 282-289.

62. Snietura M, Jaworska M, Mlynarczyk-Liszka J, et al. PTEN as a prognostic and predictive marker in postoperative radiotherapy for squamous cell cancer of the head and neck. PLoS One 2012; 7: e33396.

63. Ahmed MW, Kayani MA, Shabbir G, et al. Expression of PTEN and its correlation with proliferation marker Ki-67 in head and neck cancer. Int J Biol Markers 2016; 31: e193-203.

64. Turk A, Garcia-Carracedo D, Kent D, et al. Stathmin as a surrogate marker of phosphatidylinositol-3-kinase pathway activity: Towards precision medicine in HPV-negative head \& neck squamous cell carcinoma. Genes \& Diseases 2020; doi: 10.1016/ j.gendis.2020.12.002.

\section{Address for correspondence}

\section{Anna Janecka-Widła}

Department of Tumor Pathology

Maria Sklodowska-Curie National Research Institute of Oncology

Krakow Branch, Poland

Garncarska 11

31-115 Krakow, Poland

Tel. +48 126348410

e-mail: anna.janecka@onkologia.krakow.pl 